\title{
A rainbow blow-up lemma for almost optimally bounded edge-colourings
}

\author{
Stefan Ehard ${ }^{1}$, Stefan Glock ${ }^{2}$ and Felix Joos ${ }^{(\mathbb{D}} 3$ \\ ${ }^{1}$ Institut für Optimierung und Operations Research, Universität Ulm, Germany; E-mail: stefan.ehard@uni-ulm.de. \\ ${ }^{2}$ Institute for Theoretical Studies, ETH Zürich, Switzerland; E-mail: stefan.glock@eth-its.ethz.ch. \\ ${ }^{3}$ Institut für Informatik, Universität Heidelberg, Germany; E-mail: joos@informatik.uni-heidelberg.de.
}

Received: 21 August 2019; Revised: 12 March 2020; Accepted: 16 June 2020

2020 Mathematics Subject Classification: Primary - 05C15; Secondary - 05C35, 05C51, 05C60, 05C70, 05C78, 05B40

Keywords: blow-up lemma; rainbow colourings

\begin{abstract}
A subgraph of an edge-coloured graph is called rainbow if all its edges have different colours. We prove a rainbow version of the blow-up lemma of Komlós, Sárközy, and Szemerédi that applies to almost optimally bounded colourings. A corollary of this is that there exists a rainbow copy of any bounded-degree spanning subgraph $H$ in a quasirandom host graph $G$, assuming that the edge-colouring of $G$ fulfills a boundedness condition that is asymptotically best possible.

This has many applications beyond rainbow colourings: for example, to graph decompositions, orthogonal double covers, and graph labellings.
\end{abstract}

\section{Contents}

1 Introduction 1

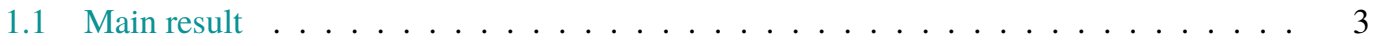

2 Applications $\quad 4$

3 Proof overview $\quad 7$

4 Preliminaries $\quad 8$

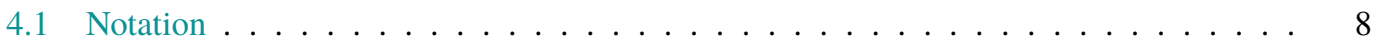

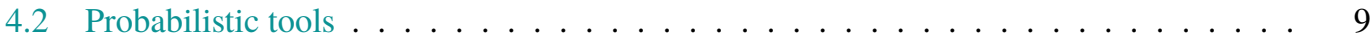

4.3 Pseudorandom hypergraph matchings . . . . . . . . . . . . . . . . 9

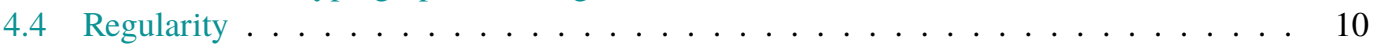

4.5 Another rainbow blow-up lemma . . . . . . . . . . . . . . 10

5 Colour splitting $\quad 11$

6 Approximate Embedding Lemma $\quad 17$

7 Proof of Lemma 1.4 $\quad 24$

\section{Introduction}

We study rainbow embeddings of bounded-degree spanning subgraphs into quasirandom graphs with almost optimally bounded edge-colourings. Moreover, following the recent work of Montgomery,

(C) The Author(s), 2020. Published by Cambridge University Press. This is an Open Access article, distributed under the terms of the Creative Commons Attribution licence (http://creativecommons.org/licenses/by/4.0/), which permits unrestricted re-use, distribution, and reproduction in any medium, provided the original work is properly cited. 
Pokrovskiy, and Sudakov [40] on embedding rainbow trees, we present several applications to graph decompositions, graph labellings, and orthogonal double covers.

Given a (not necessarily proper) edge-colouring of a graph, a subgraph is called rainbow if all its edges have different colours. Rainbow colourings appear in many different contexts of combinatorics, and many problems beyond graph colouring can be translated into a rainbow subgraph problem. What makes this concept so versatile is that it can be used to find 'conflict-free' subgraphs. More precisely, an edge-colouring of a graph $G$ can be interpreted as a system of conflicts on $E(G)$, where two edges conflict if they have the same colour. A subgraph is then conflict-free if and only if it is rainbow. For instance, rainbow matchings in $K_{n, n}$ can be used to model transversals in Latin squares. The study of Latin squares dates back to the work of Euler in the 18th century and has since been a fascinating and fruitful area of research. The famous Ryser-Brualdi-Stein conjecture asserts that every $n \times n$ Latin square has a partial transversal of size $n-1$, which is equivalent to saying that any proper $n$-edge-colouring of $K_{n, n}$ admits a rainbow matching of size $n-1$.

As a second example, we consider a powerful application of rainbow colourings to graph decompositions. Graph decomposition problems are central problems in graph theory with a long history, and many fundamental questions are still unanswered. We say that $H_{1}, \ldots, H_{t}$ decompose $G$ if $H_{1}, \ldots, H_{t}$ are edge-disjoint subgraphs of $G$ covering every edge of $G$. Perhaps one of the oldest decomposition results is Walecki's theorem from 1892 saying that $K_{2 n+1}$ can be decomposed into Hamilton cycles. His construction gives not only any decomposition but also a 'cyclic' decomposition based on a rotation technique, by finding one Hamilton cycle $H^{*}$ in $K_{2 n+1}$ and a permutation $\pi$ on $V\left(K_{2 n+1}\right)$ such that the permuted copies $\pi^{i}\left(H^{*}\right)$ of $H^{*}$ for $i=0, \ldots, n-1$ are pairwise edge-disjoint (and thus decompose $\left.K_{2 n+1}\right)$. The difficulty here is, of course, finding $H^{*}$ given $\pi$, or vice versa. Unfortunately, for many other decomposition problems, this is not as easy, or indeed not possible at all. In recent years, some exciting progress has been made in the area of (hyper-)graph decompositions: for example, Keevash's proof of the Existence conjecture [26] and generalizations thereof [19, 27], progress on the Gyárfás-Lehel treepacking conjecture [3, 24], and the resolution of the Oberwolfach problem [18]. Those results are based on very different techniques, such as absorbing-type methods, randomised constructions, and variations of Szemerédi's regularity technique. In a recent paper, Montgomery, Pokrovskiy, and Sudakov [40] brought the use of the rotation technique back into focus when proving an old conjecture of Ringel approximately, by reducing it to a rainbow embedding problem. A similar approach has previously been used by Drmota and Lladó [11] in connection with a bipartite version of Ringel's conjecture posed by Graham and Häggkvist. Ringel conjectured in 1963 that $K_{2 n+1}$ can be decomposed into $2 n+1$ copies of any given tree with $n$ edges. A strengthening of Ringel's conjecture is due to Kotzig [34], who conjectured in 1973 that there even exists a cyclic decomposition. This can be phrased as a rainbow embedding problem as follows: order the vertices of $K_{2 n+1}$ cyclically, and colour each edge $\{i, j\} \in E\left(K_{2 n+1}\right)$ with its distance (that is, the distance of $i, j$ in the cyclic ordering), which is a number between 1 and $n$. The simple but crucial observation is that if $T$ is a rainbow subtree, then $T$ can be rotated according to the cyclic vertex ordering, yielding $2 n+1$ edge-disjoint copies of $T$ (and thus a cyclic decomposition if $T$ has $n$ edges). Note that for each vertex $v$ and any given distance, there are only two vertices that have exactly this distance from $v$. More generally, an edge-colouring is called locally $k$-bounded if each colour class has maximum degree at most $k$. The following statement thus implies Kotzig's and Ringel's conjecture: any locally 2-bounded edge-colouring of $K_{2 n+1}$ contains a rainbow copy of any tree with $n$ edges. Montgomery, Pokrovskiy, and Sudakov [40] proved the following asymptotic version of this statement, which in turn yields asymptotic versions of these conjectures (all asymptotic terms are considered as $n \rightarrow \infty$ ).

Theorem 1.1 ([40]). For fixed $k$, any locally $k$-bounded edge-colouring of $K_{n}$ contains a rainbow copy of any tree with $(1-o(1)) n / k$ edges.

Our main results are very similar in spirit. Roughly speaking, instead of dealing with trees, our results apply to general graphs $H$, but we require $H$ to have bounded degree, whereas one of the great achievements of [40] is that no such requirement is necessary when dealing with trees. The following is a special case of our main result (Theorem 1.3). An edge-colouring is called (globally) $k$-bounded if any colour appears at most $k$ times. 
Theorem 1.2. Suppose $H$ is a graph on at most $n$ vertices with $\Delta(H)=\mathcal{O}(1)$. Then any locally $\mathcal{O}(1)$ bounded and globally $(1-o(1))\left(\begin{array}{l}n \\ 2\end{array}\right) / e(H)$-bounded edge-colouring of $K_{n}$ contains a rainbow copy of $H$.

It is plain that any locally $k$-bounded colouring is (globally) $k n / 2$-bounded. Thus, Theorem 1.2 implies Theorem 1.1 for bounded-degree trees. Note that the assumption that the colouring is $(1-o(1))\left(\begin{array}{l}n \\ 2\end{array}\right) / e(H)$-bounded is asymptotically best possible in the sense that if the colouring was not $\left(\begin{array}{l}n \\ 2\end{array}\right) / e(H)$-bounded, there might be fewer than $e(H)$ colours, making the existence of a rainbow copy of $H$ impossible.

Beyond the approximate solution of Ringel's conjecture, Montgomery, Pokrovskiy, and Sudakov also provide applications of their result to graph labelling and orthogonal double covers. Our applications are very much inspired by theirs and are essentially proved analogously. We refer the discussion of these applications to Section 2. We also note that since the initial submission of this manuscript, Montgomery, Pokrovskiy, and Sudakov [39] have proved Ringel's and Kotzig's conjecture exactly for all sufficiently large $n$.

Rainbow embedding problems have also been extensively studied for their own sake. For instance, Erdôs and Stein asked for the maximal $k$ such that any $k$-bounded edge-colouring of $K_{n}$ contains a rainbow Hamilton cycle (see also [15]). After several subsequent improvements, Albert, Frieze, and Reed [2] showed that $k=\Omega(n)$. Theorem 1.2 implies that under the additional assumption that the colouring is locally $\mathcal{O}(1)$-bounded, we have $k=(1-o(1)) n / 2$, which is essentially best possible. This is not a new result but also follows from results in [28, 38]. However, the results in [28, 38] are limited to finding Hamilton cycles or $F$-factors (in fact, approximate decompositions into these structures). Theorem 1.2 allows the same conclusion if we seek a $\sqrt{n / 2} \times \sqrt{n / 2}$ grid, say, or any other boundeddegree graph with roughly $n$ edges. For general subgraphs $H$, the best previous result is due to Böttcher, Kohayakawa, and Procacci [6], who showed that given any $n /\left(51 \Delta^{2}\right)$-bounded edge-colouring of $K_{n}$ and any graph $H$ on $n$ vertices with $\Delta(H) \leq \Delta$, one can find a rainbow copy of $H$. Our Theorem 1.2 improves, for bounded-degree graphs, the global boundedness condition to an asymptotically best possible one, under the additional assumption that the colouring is locally $\mathcal{O}(1)$-bounded.

\subsection{Main result}

We now state a more general version of Theorem 1.2. We say that a graph $G$ on $n$ vertices is $(\varepsilon, d)$ quasirandom if for all $v \in V(G)$, we have $\operatorname{deg}_{G}(v)=(d \pm \varepsilon) n$, and for all disjoint $S, T \subseteq V(G)$ with $|S|,|T| \geq \varepsilon$, we have $e_{G}(S, T)=(d \pm \varepsilon)|S||T|$.

Theorem 1.3. For all $d, \gamma \in(0,1]$ and $\Delta, \Lambda \in \mathbb{N}$, there exist $\varepsilon>0$ and $n_{0} \in \mathbb{N}$ such that the following holds for all $n \geq n_{0}$. Suppose $G$ and $H$ are graphs on $n$ vertices, $G$ is $(\varepsilon, d)$-quasirandom, and $\Delta(H) \leq \Delta$. Then given any locally $\Lambda$-bounded and globally $(1-\gamma) e(G) / e(H)$-bounded edge-colouring of $G$, there is a rainbow copy of $H$ in $G$.

Clearly, Theorem 1.3 implies Theorem 1.2. We derive Theorem 1.3 from an even more general 'blow-up lemma' (Lemma 1.4). The original blow-up lemma of Komlós, Sárközy, and Szemerédi [30], developed roughly 20 years ago, is a powerful tool to find spanning subgraphs and has numerous important applications in extremal combinatorics [8, 18, 31, 32, 33, 35, 36]. Roughly speaking, it says that given a $k$-partite graph $G$ that is 'super-regular' between any two vertex classes, and a $k$-partite bounded-degree graph $H$ with a matching vertex partition, then $H$ is a subgraph of $G$. Note that the conclusion is trivial if $G$ is complete $k$-partite, so the crux here is that instead of requiring $G$ to be complete between any two vertex classes, super-regularity suffices. Such a scenario can often be obtained in conjunction with Szemerédi's regularity lemma, which makes it widely applicable. Many variations of the blow-up lemma have been obtained over the years (for example, [4, 7, 10, 25, 29, 42]). Recently, Joos and Glock [17] proved a rainbow blow-up lemma for $o(n)$-bounded edge-colourings that allows to find a rainbow embedding of $H$. The present paper builds upon this result. The key novelty is that instead of requiring the colouring to be $o(n)$-bounded, our new result applies for almost optimally bounded 
colourings. (But we assume here that the colouring is locally $\mathcal{O}(1)$-bounded, which is not necessary in [17].)

To state our new rainbow blow-up lemma, we need to introduce some terminology. If $c: E(G) \rightarrow C$ is an edge-colouring of a graph $G$ and $\alpha \in C$, denote by $e^{\alpha}(G)$ the number of $\alpha$-coloured edges of $G$. Moreover, for disjoint $S, T \subseteq V(G)$, denote by $e_{G}^{\alpha}(S, T)$ the number of $\alpha$-coloured edges of $G$ with one endpoint in $S$ and the other one in $T$. Define $d_{G}(S, T):=e_{G}(S, T) /|S||T|$ as the density of the pair $S, T$ in $G$. We say that the bipartite graph $G$ with vertex classes $\left(V_{1}, V_{2}\right)$ is $(\varepsilon, d)$-super-regular if

○ For all $S \subseteq V_{1}$ and $T \subseteq V_{2}$ with $|S| \geq \varepsilon\left|V_{1}\right|,|T| \geq \varepsilon\left|V_{2}\right|$, we have $d_{G}(S, T)=d \pm \varepsilon$.

○ For all $i \in[2]$ and $v \in V_{i}$, we have $\operatorname{deg}_{G}(v)=(d \pm \varepsilon)\left|V_{3-i}\right|$.

We say that $\left(H, G,\left(X_{i}\right)_{i \in[r]},\left(V_{i}\right)_{i \in[r]}\right)$ is an $(\varepsilon, d)$-super-regular blow-up instance if

- $H$ and $G$ are graphs, $\left(X_{i}\right)_{i \in[r]}$ is a partition of $V(H)$ into independent sets, $\left(V_{i}\right)_{i \in[r]}$ is a partition of $V(G)$, and $\left|X_{i}\right|=\left|V_{i}\right|$ for all $i \in[r]$, and

○ For all $i j \in\left(\begin{array}{c}{[r]} \\ 2\end{array}\right)$, the bipartite graph $G\left[V_{i}, V_{j}\right]$ is $(\varepsilon, d)$-super-regular.

We say that $\phi: V(H) \rightarrow V(G)$ is an embedding of $H$ into $G$ if $\phi$ is injective and $\phi(x) \phi(y) \in E(G)$ for all $x y \in E(H)$. We also write $\phi: H \rightarrow G$ in this case. We say that $\phi$ is rainbow if $\phi(H)$ is rainbow.

We now state our new rainbow blow-up lemma.

Lemma 1.4 (Rainbow blow-up lemma). For all $d, \gamma \in(0,1]$ and $\Delta, \Lambda, r \in \mathbb{N}$, there exist $\varepsilon>0$ and $n_{0} \in \mathbb{N}$ such that the following holds for all $n \geq n_{0}$. Suppose $\left(H, G,\left(X_{i}\right)_{i \in[r]},\left(V_{i}\right)_{i \in[r]}\right)$ is an $(\varepsilon, d)$ super-regular blow-up instance. Assume further that

(i) $\Delta(H) \leq \Delta$.

(ii) $\left|V_{i}\right|=(1 \pm \varepsilon) n$ for all $i \in[r]$.

(iii) $c: E(G) \rightarrow C$ is a locally $\Lambda$-bounded edge-colouring such that the following holds for all $\alpha \in C$ :

$$
\sum_{i j \in\left(\begin{array}{c}
{[r]} \\
2
\end{array}\right)} e_{G}^{\alpha}\left(V_{i}, V_{j}\right) e_{H}\left(X_{i}, X_{j}\right) \leq(1-\gamma) d n^{2} .
$$

Then there exists a rainbow embedding $\phi$ of $H$ into $G$ such that $\phi(x) \in V_{i}$ for all $i \in[r]$ and $x \in X_{i}$.

The boundedness condition in (iii) can often be simplified, for instance in the following natural situations: if $e_{H}\left(X_{i}, X_{j}\right)$ is the same for all pairs $i, j$, then $c$ needs to be $(1-\gamma) e\left(G\left[V_{1}, \ldots, V_{r}\right]\right) / e(H)$ bounded. Similarly, if $c$ is 'colour-split'-that is, $e_{G}^{\alpha}\left(V_{i}, V_{j}\right) \in\left\{e^{\alpha}(G), 0\right\}$-then $c$ needs to be $(1-\gamma) e\left(G\left[V_{i}, V_{j}\right]\right) / e\left(H\left[X_{i}, X_{j}\right]\right)$-bounded for all $i j \in\left(\begin{array}{c}{[r]} \\ 2\end{array}\right)$. Both conditions are easily seen to be asymptotically best possible. Condition (iii) is designed to work in the general setting of Lemma 1.4. In fact, we will deduce Lemma 1.4 in Section 7 from a reduced instance (Lemma 7.1) where the colouring $c$ is colour-split. In the proof of Theorem 1.3, we will randomly partition $V(G)$ into equal-sized $\left(V_{i}\right)_{i \in[r]}$ and see that (iii) holds.

\section{Applications}

In this section, we discuss applications of our main result to graph decompositions, graph labelling, and orthogonal double covers. As mentioned before, these applications are inspired by recent work of Montgomery, Pokrovskiy, and Sudakov [40] and basically transfer their applications from trees to general, yet bounded degree, graphs.

\section{Graph decompositions}

We briefly explain the general idea of utilizing rainbow edge-colourings to find graph decompositions and then give two examples. 
Suppose $G$ is a graph and $\Gamma$ is a subgroup of the automorphism group $A u t(G)$. If for some subgraph $H$ of $G,\{\phi(H)\}_{\phi \in \Gamma}$ is a collection of edge-disjoint subgraphs of $G$, we call this a $\Gamma$-generated $H$-packing in $G$; and if every edge of $G$ is covered, then it is a $\Gamma$-generated $H$-decomposition of $G$. For instance, in Walecki's theorem, $G$ is the complete graph and $\Gamma$ is generated by one permutation $\pi$. We say that a packing/decomposition of $K_{n}$ is cyclic if $\Gamma$ is isomorphic to $\mathbb{Z}_{n}$. Recall Kotzig's conjecture that for any given tree $T$ with $n$ edges, there exists a cyclic $T$-decomposition of $K_{2 n+1}$. Note that there are two natural divisibility conditions for the existence of such a decomposition: one 'global' edge divisibility condition and one 'local' degree condition. First, the number of edges of $K_{2 n+1}$ is $(2 n+1) n$, which is divisible by $n$. Second, every vertex of $K_{2 n+1}$ is supposed to play the role of every vertex of $T$ exactly once, and thus we need that $\sum_{v \in V(T)} d_{T}(v)=2 n$, which is true by the hand-shaking lemma. However, note that we have not used the fact that $T$ is a tree. The same divisibility conditions hold for any graph with $n$ edges. We thus propose the following conjecture as an analogue to Kotzig's conjecture for general (bounded degree) graphs.

Conjecture 2.1. For all $\Delta \in \mathbb{N}$, there exists $n_{0}$ such that for all $n \geq n_{0}$, the following is true. For any graph $H$ with $n$ edges and $\Delta(H) \leq \Delta$, there exists a cyclic $H$-decomposition of $K_{2 n+1}$.

We will provide some evidence for this conjecture below (Theorem 2.3). First, we discuss in a general way how to use rainbow embeddings to find $\Gamma$-generated packings and decompositions. Let $G$ and $\Gamma$ be as above. Then $\Gamma$ acts on $G$ as a group action, and every element $\phi \in \Gamma$ sends vertices onto vertices and edges onto edges. The orbit $\Gamma \cdot e$ of an edge $e$ is defined as $\Gamma \cdot e:=\{\phi(e): \phi \in \Gamma\}$. It is well known that two orbits are either disjoint or equal. Hence we may colour the edges of $G$ according to which orbit they belong to. We refer to the orbit colouring $c_{o}^{\Gamma}$ of $G$ induced by $\Gamma$ and define $c_{o}^{\Gamma}(e):=\Gamma \cdot e$ for all $e \in E(G)$.

The following simple lemma now asserts that if we can find a rainbow copy with respect to the orbit colouring, and all orbits have maximum size, then the copies of $H$ obtained via $\Gamma$ are pairwise edge-disjoint. The proof is immediate and thus omitted.

Lemma 2.2. Let $G$ be a graph, and let $\Gamma$ be a subgroup of Aut $(G)$ such that $|\Gamma \cdot e|=|\Gamma|$ for all $e \in E(G)$. Suppose that $H$ is a rainbow subgraph in $G$ with respect to $c_{o}^{\Gamma}$. Then $\{\phi(H)\}_{\phi \in \Gamma}$ is a $\Gamma$-generated H-packing in $G$.

In particular, if $|\Gamma|=e(G) / e(H)$, then this yields a $\Gamma$-generated $H$-decomposition of $G$.

Theorem 2.3. For all $\Delta \in \mathbb{N}$, there exist $\varepsilon>0$ and $n_{0} \in \mathbb{N}$ such that the following holds for all $n \geq n_{0}$. Suppose $H$ is a graph with $|V(H)| \leq n, \Delta(H) \leq \Delta$ and at most $(1-\varepsilon) n / 2$ edges. Then $K_{n}$ contains a cyclic H-packing.

Proof. Let $G$ be the graph on vertex set $[n]$ that is the complete graph if $n$ is odd and is otherwise obtained from the complete graph by deleting the edges $\{i, i+n / 2\}$ for all $i \in[n / 2]$. Consider the subgroup $\Gamma$ of $\operatorname{Aut}(G)$ that is generated by the automorphism that sends a vertex $i$ to $i+1$ (modulo $n$ ). Clearly, $\Gamma \cong \mathbb{Z}_{n}$, and hence $|\Gamma|=n$. In addition, $|\Gamma \cdot e|=n$ for all $e \in E(G)$, and $c_{o}^{\Gamma}$ is locally 2-bounded. Therefore, Theorem 1.3 yields a rainbow copy of $H$ with respect to $c_{o}^{\Gamma}$ in $G$, which by Lemma 2.2 yields a cyclic $H$-packing in $G \subseteq K_{n}$.

We can also deduce a partite version of this. For simplicity, we consider only the bipartite case.

Theorem 2.4. For all $\Delta \in \mathbb{N}$, there exist $\varepsilon>0$ and $n_{0} \in \mathbb{N}$ such that the following holds for all $n \geq n_{0}$. Suppose $H$ is a graph with $\Delta(H) \leq \Delta$ and at most $(1-\varepsilon) n$ edges, and $V(H)$ is partitioned into 2 independent sets of size $n$. Then the complete bipartite graph $K_{n, n}$ contains a $\mathbb{Z}_{n}$-generated $H$-packing.

Proof. We proceed similarly as in Theorem 2.3. Let $K_{n, n}$ have vertex set $\{(1, i),(2, i)\} i \in[n]$ and edge set $\{(1, i)(2, j)\} i, j \in[n]$. Consider the subgroup $\Gamma$ of $A u t(G)$ that is generated by the automorphism that sends each vertex $(\ell, i)$ to $(\ell, i+1)$ (modulo $n$ in the second coordinate) for $\ell \in[2]$. Consequently, $\Gamma \cong \mathbb{Z}_{n}$. Moreover, $|\Gamma \cdot e|=n$ for all $e \in E\left(K_{n, n}\right)$, and $c_{o}^{\Gamma}$ is proper. Thus, Lemma 1.4 yields a rainbow copy of $H$ in $K_{n, n}$ with respect to $c_{o}^{\Gamma}$. Then Lemma 2.2 completes the proof. 
These results demonstrate the usefulness of rainbow embeddings to decomposition problems. Clearly, the application is limited to decompositions of a host graph into copies of the same graph $H$. Approximate decomposition results that do not arise from a group action but from random procedures have been studied recently in great depth. At the expense that one does not obtain very symmetric (approximate) decompositions, it is possible to embed different graphs and not only many copies of a single graph. In particular, the blow-up lemma for approximate decompositions by Kim, Kühn, Osthus, and Tyomkyn [29] yields approximate decompositions into bounded degree graphs of quasirandom multipartite graphs. Both this and another recent result of Allen, Böttcher, Hladký, and Piguet [5] imply Conjecture 2.1 asymptotically for non-cyclic decompositions.

\section{Orthogonal double covers}

An orthogonal double cover of $K_{n}$ by some graph $F$ is a collection of $n$ copies of $F$ in $K_{n}$ such that every edge of $K_{n}$ is contained in exactly two copies, and each two copies have exactly one edge in common. Note that $F$ must have exactly $n-1$ edges. For instance, an orthogonal double cover of $K_{\left(\begin{array}{c}k \\ 2\end{array}\right)+1}$ by $K_{k}$ is equivalent to a biplane, which is, roughly speaking, the orthogonal double cover version of a finite projective plane. Only a handful of such biplanes are known, and it is a major open question whether there are infinitely many.

Another natural candidate for $F$ is a spanning tree. Gronau, Mullin, and Rosa conjectured the following.

Conjecture 2.5 (Gronau, Mullin, Rosa [21]). Let $T$ be an arbitrary tree with $n$ vertices, $n \geq 2$, where $T$ is not the path of length 3 . Then there exists an orthogonal double cover of $K_{n}$ by $T$.

Montgomery, Pokrovskiy, and Sudakov [40] proved an asymptotic version of this when $n$ is a power of 2, using their Theorem 1.1. Similarly, our main theorem yields approximate orthogonal double covers by copies of any bounded degree graph with $(1-o(1)) n$ edges whenever $n$ is a power of 2 . We omit the proof as it is verbatim the same as in [40].

Theorem 2.6. For all $\Delta \in \mathbb{N}$, there exist $\varepsilon>0$ and $n_{0} \in \mathbb{N}$ such that the following holds for all $n \geq n_{0}$ with $n=2^{k}$ for some $k \in \mathbb{N}$. Suppose $H$ is a graph with $|V(H)| \leq n, \Delta(H) \leq \Delta$ and at most $(1-\varepsilon) n$ edges. Then the complete graph $K_{n}$ contains $n$ copies of $H$ such that every edge of $K_{n}$ belongs to at most two copies, and any two copies have at most one edge in common.

\section{Graph labellings}

The study of graph labellings began in the 1960s and has since produced a vast amount of different concepts, results, and applications (see, for example, the survey [16]). Perhaps the most popular types of labellings are graceful labellings and harmonious labellings. The former were introduced by Rosa [43] in 1967. Given a graph $H$ with $q$ edges, a graceful labelling of $H$ is an injection $f: V(H) \rightarrow[q+1]$ such that the induced edge labels $|f(x)-f(y)|, x y \in E(H)$ are pairwise distinct, and $H$ is graceful if such a labelling exists. The graceful tree conjecture asserts that all trees are graceful. Rosa [43] showed that this would imply the aforementioned Ringel-Kotzig conjecture. Despite extensive research, this conjecture remains wide open. Adamaszek, Allen, Grosu, and Hladký [1] recently proved that almost all trees are almost graceful.

Harmonious labellings were introduced by Graham and Sloane [20] in 1980. Given a graph $H$ and an abelian group $\Gamma$, a $\Gamma$-harmonious labelling of $H$ is an injective map $f: V(H) \rightarrow \Gamma$ such that the induced edge labels $f(x)+f(y), x y \in E(H)$ are pairwise distinct, and $H$ is $\Gamma$-harmonious if such a labelling exists. Graham and Sloane asked which graphs $H$ are $\mathbb{Z}_{e(H)}$-harmonious. Note that this necessitates that $|V(H)| \leq e(H)$. In the special case when $H$ is a tree on $n$ vertices, they conjectured that there exists an injective map $f: V(H) \rightarrow[n]$ such that the induced edge labels $f(x)+f(y), x y \in E(H)$ are pairwise distinct modulo $n-1$. Żak [44] proposed a weakening of this. He conjectured that every 
tree on $n-o(n)$ vertices is $\mathbb{Z}_{n}$-harmonious. Montgomery, Pokrovskiy, and Sudakov [40] proved Żak's conjecture as a corollary of Theorem 1.1. Using our Theorem 1.2, we can deduce a similar statement for general bounded degree graphs.

Theorem 2.7. For all $\Delta \in \mathbb{N}$, there exist $\varepsilon>0$ and $n_{0} \in \mathbb{N}$ such that the following holds for all $n \geq n_{0}$. Suppose $H$ is a graph with at most $n$ vertices, at most $(1-\varepsilon) n$ edges, and $\Delta(H) \leq \Delta$. Let $\Gamma$ be an abelian group of order $n$. Then $H$ is $\Gamma$-harmonious.

Proof. Consider the complete graph $K_{\Gamma}$ on $\Gamma$. Define the edge-colouring $c: E\left(K_{\Gamma}\right) \rightarrow \Gamma$ by setting $c(i j)=i+j$, and note that $c$ is proper and thus $n / 2$-bounded. Hence, by Theorem $1.2, K_{\Gamma}$ contains a rainbow copy of $H$, which corresponds to a $\Gamma$-harmonious labelling of $H$.

\section{Proof overview}

In the literature, there are two common approaches for proving blow-up lemmas. The original approach of Komlós, Sárközy, and Szemerédi consists of a randomised sequential embedding algorithm, which embeds the bulk of the vertices one by one, choosing each time a random image from all available ones. This strategy has also been used in $[4,7,10,25]$.

Shortly after the appearance of the blow-up lemma, Rödl and Ruciński [42] developed an alternative proof, where instead of embedding vertices one by one, the algorithm consists of only a constant number of steps. In the $i$ th step, the whole cluster $X_{i}$ is embedded into $V_{i}$. The desired bijection is obtained as a perfect matching within a 'candidacy graph' $A_{i}$, which is an auxiliary bipartite graph between $X_{i}$ and $V_{i}$ where $x v \in E\left(A_{i}\right)$ only if $v$ is still a suitable image for $x$. Although these candidacy graphs (of clusters not yet embedded) become sparser after each step, Rödl and Ruciński were able to show that one can maintain their super-regularity throughout the procedure. This approach was also employed in [29] to prove a blow-up lemma for approximate decompositions and in [17] to prove a rainbow blow-up lemma for $o(n)$-bounded colourings, and it also underpins our proof here.

For simplicity, we consider here the following setup. Suppose $V(H)$ is partitioned into independent sets $X_{1}, X_{2}, X_{3}$ of size $n$, and $H$ consists of a perfect matching between $X_{1}$ and $X_{2}$ and a perfect matching between $X_{2}$ and $X_{3}$.

Suppose that we have already found an embedding $\phi_{1}: X_{1} \rightarrow V_{1}$, and next we want to embed $X_{2}$ into $V_{2}$. We define the bipartite graph $A_{2}$ between $X_{2}$ and $V_{2}$ by adding the edge $x v$ if $\phi_{1}(y) v \in E(G)$, where $y$ is the $H$-neighbour of $x$ in $X_{1}$. Now, the aim is to find a perfect matching $\sigma$ in $A_{2}$. Note that any such perfect matching yields a valid embedding of $H\left[X_{1}, X_{2}\right]$ into $G\left[V_{1}, V_{2}\right]$. Moreover, if we aim to find a rainbow embedding, this can be achieved as follows. For each $x v \in E\left(A_{2}\right)$, we colour $x v$ with the colour of $\phi_{1}(y) v$. Observe that if $\sigma$ is rainbow, then the embedding of $H\left[X_{1}, X_{2}\right]$ into $G\left[V_{1}, V_{2}\right]$ will be rainbow, too. Let us assume that $A_{2}$ is super-regular. It is well known that $A_{2}$ then has a perfect matching. One key ingredient in [17] was to combine this fact with a recent result of Coulson and Perarnau [9], based on the switching method, to even find a rainbow perfect matching. Unfortunately, the switching method relies upon the fact that the given colouring is $o(n)$-bounded and is thus not applicable in the present setting. Two key insights will allow us to deal with almost optimally bounded colourings.

First, note that given a proper colouring of a graph $G$, if we take a random subset $U$ of size $\mu|G|$, then with high probability, the colouring induced on $U$ will be $(1+o(1)) \mu|U|$-bounded, and thus the rainbow blow-up lemma from [17] is applicable (on $U$ ). This gives hope to combine this with an 'approximate result' on $V(G) \backslash U$ to obtain the desired embedding. Such a combination of techniques has already been successfully used in [28]. In our simplified discussion, let us thus assume we do not need to find a perfect rainbow matching $\sigma$ but would be content if $\sigma$ is almost perfect.

This leads us to the second main ingredient of our proof - matchings in hypergraphs. Given our candidacy graph $A_{2}$ and its (auxiliary) colouring $c_{2}: E\left(A_{2}\right) \rightarrow C_{2}$, we define a hypergraph $\mathcal{H}$ on $X_{2} \cup V_{2} \cup C_{2}$ where for every edge $e \in E\left(A_{2}\right)$, we add the hyperedge $e \cup\{c(e)\}$ to $\mathcal{H}$. A simple but crucial observation is that there is a one-to-one correspondence between matchings in $\mathcal{H}$ and rainbow 
matchings in $A_{2}$. In particular, a matching $\mathcal{M}$ in $\mathcal{H}$ that covers almost all vertices of $X_{2} \cup V_{2}$ would translate into our desired almost-perfect rainbow matching $\sigma$ in $A_{2}$. Here, we can make use of the rich theory of matchings in hypergraphs with small codegrees, which we will discuss in more detail in Section 4.3. At this point, we remark that since $A_{2}$ is super-regular, all vertices of $X_{2} \cup V_{2}$ have roughly the same degree in $\mathcal{H}$; and if the degrees of the colours are not larger (that is, the colouring is appropriately bounded), this will suffice to find the desired matching in $\mathcal{H}$.

Moreover, note that we assumed that $A_{2}$ is super-regular and its colouring is appropriately bounded. After embedding $X_{2}$ according to $\sigma$, we have to update the candidacy graph $A_{3}$ as we updated $A_{2}$ after embedding $X_{1}$. Of course, whether $A_{3}$ will be super-regular and its colouring appropriately bounded depends heavily on $\sigma$. For the embedding not to get stuck, we need to find in $A_{2}$ not just any $\sigma$, but a good one. To achieve this, we make use of a general hypergraph matching theorem (Theorem 4.3) proved recently by the authors in [13], which guarantees a matching $\mathcal{M}$ in $\mathcal{H}$ that is in many ways 'random-like'. This will allow us to find an almost-perfect rainbow matching $\sigma$ for which the updated candidacy graph $A_{3}$ will have the desired properties. In more detail, the conclusion of Theorem 4.3 allows to put weight functions on the edges of the hypergraph $\mathcal{H}$ and guarantees a matching $\mathcal{N}$ in $\mathcal{H}$ such that the weight covered by $\mathcal{M}$ is what we would expect from an idealized random matching (see Section 4.3 for more details). We employ such weight functions to provide that $A_{3}$ will still be super-regular and its colouring appropriately bounded. This is done in Section 6, where we prove an 'Approximate Embedding Lemma' (Lemma 6.3). As discussed, in the end, we will make use of the rainbow blow-up lemma for $o(n)$ bounded edge-colourings from [17] to turn an approximate embedding into a complete one.

This simplified setup already presents the main ingredients for the proof of our rainbow blow-up lemma (Lemma 1.4). An important step in the approach of Rödl and Ruciński [42] is to refine the partition of $H$ such that $H$ only induces matchings between its refined partition classes using the Hajnal-Szemerédi theorem. We follow the same strategy and additionally find a subgraph $G^{\prime}$ of $G$ such that the edge-colouring of $G^{\prime}$ is colour-split: that is, each colour only appears between one bipartite pair of the refined partition classes of $G^{\prime}$. This enables us to deduce Lemma 1.4 from a reduced instance (Lemma 7.1) where we impose that $H$ only induces matchings between its partition classes, and the edge-colouring of $G$ is colour-split. The main tools to perform these reductions are given in Section 5 .

\section{Preliminaries}

\subsection{Notation}

For $k \in \mathbb{N}$, we write $[k]_{0}:=[k] \cup\{0\}=\{0,1, \ldots, k\}$, where $[0]=\emptyset$. For a finite set $S$ and $i \in \mathbb{N}$, we write $\left(\begin{array}{c}S \\ i\end{array}\right)$ for the set of all subsets of $S$ of size $i$ and $2^{S}$ for the powerset of $S$. For a set $\{i, j\}$, we sometimes simply write $i j$. For $a, b, c \in \mathbb{R}$, we write $a=b \pm c$ whenever $a \in[b-c, b+c]$. For $a, b, c \in(0,1]$, we sometimes write $a \ll b \ll c$ in our statements, meaning there are increasing functions $f, g:(0,1] \rightarrow(0,1]$ such that whenever $a \leq f(b)$ and $b \leq g(c)$, then the subsequent result holds.

We only consider finite, simple, and undirected graphs. For a graph $G$, we let $V(G)$ and $E(G)$ denote the vertex set and edge set, respectively. We say $u \in V(G)$ is a $G$-neighbour of $v \in V(G)$ if $u v \in E(G)$. As usual, $\Delta(G)$ denotes the maximum degree of $G$. For $u, v \in V(G)$, let $N_{G}(u, v):=N_{G}(u) \cap N_{G}(v)$ denote the common neighbourhood of $u$ and $v$. For a set $S \subseteq V(G)$, let $N(S):=\bigcup_{v \in S} N_{G}(v)$. For disjoint subsets $A, B \subseteq V(G)$, let $G[A, B]$ denote the bipartite subgraph of $G$ between $A$ and $B$ and $G[A]$, the subgraph in $G$ induced by $A$. Let $e(G)$ be the number of edges of $G$, and let $e_{G}(A, B)$ denote the number of edges of $G[A, B]$. We let $G^{2}$ denote the square of $G$ : that is, the graph obtained from $G$ by adding edges between vertices that have a common neighbour in $G$. A subset $X \subseteq V(G)$ is 2-independent if it is independent in $G^{2}$.

Let $G$ be a graph. Given a set $C$, a function $c: E(G) \rightarrow 2^{C}$ is called an edge set colouring of $G$. A colour $\alpha \in C$ appears on an edge $e$ if $\alpha \in c(e)$. We define the codegree of $c$ as the maximum number of edges on which any two fixed colours appear together. For a colour $\alpha \in C$, a vertex $v \in V(G)$, and 
disjoint sets $A, B \subseteq V(G)$, we define

$\circ \operatorname{deg}_{G}^{\alpha}(v):=\mid\left\{u \in N_{G}(v): \alpha\right.$ appears on $\left.u v\right\} \mid$

○ $e_{G}^{\alpha}(A, B):=\mid\{a b \in E(G): a \in A, b \in B$, and $\alpha$ appears on $a b\} \mid$

$\circ e^{\alpha}(G):=\mid\{e \in E(G): \alpha$ appears on $e\} \mid$

We say that

- $c$ is (globally) $k$-bounded if each colour appears on at most $k$ edges.

$\circ c$ is locally $\Lambda$-bounded if each colour class has maximum degree at most $\Lambda$.

Given a partition $\left(V_{i}\right)_{i \in[r]}$ of $V(G)$, we say that $c$ is colour-split with respect to $\left(V_{i}\right)_{i \in[r]}$ if for all $e, f \in E(G)$ we have $c(e) \cap c(f)=\emptyset$ whenever $e \in E\left(G\left[V_{i}, V_{j}\right]\right)$ and $f \notin E\left(G\left[V_{i}, V_{j}\right]\right)$. If the partition is clear from the context, we just say that $c$ is colour-split. We call a subgraph $G^{\prime}$ of $G$ rainbow if all the edges in $G^{\prime}$ have pairwise disjoint colour sets.

\subsection{Probabilistic tools}

In this section, we state a well-known Chernoff-type bound and McDiarmid's inequality. These will be the main tools to establish concentration of a random variable for the large deviation results we need.

Theorem 4.1 (Chernoff's bound; see [23]). Suppose $X_{1}, \ldots, X_{m}$ are independent Bernoulli random variables. Let $X:=\sum_{i=1}^{m} X_{i}$. Then for all $t \geq 0$,

$$
\mathbb{P}[|X-\mathbb{E}[X]| \geq t] \leq 2 \exp \left(-\frac{t^{2}}{2(\mathbb{E}[X]+t / 3)}\right) .
$$

Theorem 4.2 (McDiarmid's inequality; see [37, Lemma 1.2]). Suppose $X_{1}, \ldots, X_{m}$ are independent random variables, and suppose $b_{1}, \ldots, b_{m} \in[0, B]$. Suppose $X$ is a real-valued random variable determined by $X_{1}, \ldots, X_{m}$ such that changing the outcome of $X_{i}$ changes $X$ by at most $b_{i}$ for all $i \in[m]$. Then for all $t>0$, we have

$$
\mathbb{P}[|X-\mathbb{E}[X]| \geq t] \leq 2 \exp \left(-\frac{2 t^{2}}{\sum_{i=1}^{m} b_{i}^{2}}\right) \leq 2 \exp \left(-\frac{2 t^{2}}{B \sum_{i=1}^{m} b_{i}}\right) .
$$

\subsection{Pseudorandom hypergraph matchings}

As sketched in Section 3, we use hypergraph matchings to model rainbow embeddings. In this section, we introduce a theorem from [13] on 'pseudorandom' hypergraph matchings (Theorem 4.3), which will play an important role in Section 6.

Following the seminal result of Rödl [41] on approximate Steiner systems, Pippenger observed that any almost regular uniform hypergraph with small codegrees has an almost perfect matching. In [13], the authors proved a tool that allows to obtain 'pseudorandom' matchings in this setting. To make this more precise, we define for a hypergraph $\mathcal{H}$ and vertices $u, v \in V(\mathcal{H})$ the degree $\operatorname{deg}_{\mathcal{H}}(v):=|\{e \in E(\mathcal{H}): v \in e\}|$ and codegree $\operatorname{deg}_{\mathcal{H}}(u v):=|\{e \in E(\mathcal{H}):\{u, v\} \subseteq e\}|$. We define

$$
\Delta(\mathcal{H}):=\max _{v \in V(\mathcal{H})} \operatorname{deg}_{\mathcal{H}}(v) \text { and } \Delta^{c}(\mathcal{H}):=\max _{u \neq v \in V(\mathcal{H})} \operatorname{deg}_{\mathcal{H}}(u v)
$$

to be the maximum degree and maximum codegree of $\mathcal{H}$, respectively. A matching in $\mathcal{H}$ is a collection of disjoint edges.

Suppose for simplicity that we are given a $D$-regular hypergraph and want to find an (almost) perfect matching $\mathcal{M}$. Moreover, we wish $\mathcal{M}$ to be 'pseudorandom': that is, to have certain properties that we expect from an idealized random matching. Heuristically, we may expect that every edge of 
$\mathcal{H}$ is in a random perfect matching with probability $1 / D$. Thus, given a subset $U \subseteq V(\mathcal{H})$, we expect $|E(\mathcal{H}[U])| / D$ matching edges inside $U$, and we may require similar statistics for matching edges crossing certain vertex sets. This can be formalized in a quite general way. Given a set $X$, a weight function on $X$ is a function $\omega: X \rightarrow \mathbb{R}_{\geq 0}$. For a subset $X^{\prime} \subseteq X$, we define $\omega\left(X^{\prime}\right):=\sum_{x \in X^{\prime}} \omega(x)$. If $\omega$ is a weight function on $E(\mathcal{H})$, the above heuristic would imply that we expect $\omega(\mathcal{M}) \approx \omega(E(\mathcal{H})) / D$. The following theorem asserts that a hypergraph with small codegrees has a matching that is pseudorandom in this sense.

Theorem 4.3 ([13]). Suppose $1 / \Delta \ll \delta, 1 / r$, and $r \in \mathbb{N}$ with $r \geq 2$, and let $\varepsilon:=\delta / 50 r^{2}$. Let $\mathcal{H}$ be an $r$-uniform hypergraph with $\Delta(\mathcal{H}) \leq \Delta$ and $\Delta^{c}(\mathcal{H}) \leq \Delta^{1-\delta}$ as well as $e(\mathcal{H}) \leq \exp \left(\Delta^{\varepsilon^{2}}\right)$. Suppose that $\mathcal{W}$ is a set of at most $\exp \left(\Delta^{\varepsilon^{2}}\right)$ weight functions on $E(\mathcal{H})$. Then there exists a matching $\mathcal{M}$ in $\mathcal{H}$ such that $\omega(\mathcal{M})=\left(1 \pm \Delta^{-\varepsilon}\right) \omega(E(\mathcal{H})) / \Delta$ for all $\omega \in \mathcal{W}$ with $\omega(E(\mathcal{H})) \geq \max _{e \in E(\mathcal{H})} \omega(e) \Delta^{1+\delta}$.

We refer the interested reader to [13] for more information on the preceding results and further variants and applications of Theorem 4.3. In particular, Ehard and Joos recently used a more general version of Theorem 4.3 to give a short proof of the blow-up lemma for approximate decompositions [14].

\subsection{Regularity}

Given $\varepsilon>0$ and $d \in[0,1]$, a bipartite graph $G$ with vertex classes $\left(V_{1}, V_{2}\right)$ is called $(\varepsilon, d)$-regular if for all pairs $S \subseteq V_{1}$ and $T \subseteq V_{2}$ with $|S| \geq \varepsilon\left|V_{1}\right|,|T| \geq \varepsilon\left|V_{2}\right|$, we have $d_{G}(S, T)=d \pm \varepsilon$. The following is one of the fundamental properties of $\varepsilon$-regularity.

Fact 4.4. Let $G$ be an $(\varepsilon, d)$-regular bipartite graph with partition $(A, B)$, and let $Y \subseteq B$ with $|Y| \geq \varepsilon|B|$. Then all but at most $2 \varepsilon|A|$ vertices of $A$ have $(d \pm \varepsilon)|Y|$ neighbours in $Y$.

We will also often use the fact that super-regularity is robust with respect to small vertex and edge deletions.

Fact 4.5. Suppose $1 / n \ll \varepsilon \ll d$. Let $G$ be an $(\varepsilon, d)$-super-regular bipartite graph with partition $(A, B)$, where $\varepsilon^{1 / 6} n \leq|A|,|B| \leq n$. If $\Delta(H) \leq \varepsilon n$ and $X \subseteq A \cup B$ with $|X| \leq \varepsilon n$, then $G[A \backslash X, B \backslash X]-E(H)$ is $\left(\varepsilon^{1 / 3}, d\right)$-super-regular.

The following is essentially a result from [12]. (In [12], it is proved in the case when $|A|=|B|$ with $16 \epsilon^{1 / 5}$ instead of $\epsilon^{1 / 6}$. The version stated below can be easily derived from this.)

Theorem 4.6. Suppose $1 / n \ll \varepsilon \ll \gamma, d$. Suppose $G$ is a bipartite graph with vertex partition $(A, B)$ such that $|A|=n, \gamma n \leq|B| \leq \gamma^{-1} n$ and at least $(1-5 \varepsilon) n^{2} / 2$ pairs $u, v \in A$ satisfy $\operatorname{deg}_{G}(u), \operatorname{deg}_{G}(v) \geq$ $(d-\varepsilon)|B|$, and $\left|N_{G}(u, v)\right| \leq(d+\varepsilon)^{2}|B|$. Then $G$ is $\left(\varepsilon^{1 / 6}, d\right)$-regular.

\subsection{Another rainbow blow-up lemma}

Our final tool is the following special case of the rainbow blow-up lemma from [17] for $o(n)$-bounded colourings. Even though the global boundedness condition is more restrictive there, it is still applicable on a random subset of vertices (see the discussion in Section 3). As such, it is the main tool in our proof to turn a partial rainbow embedding into a complete one.

We say that $\left(H, G,\left(X_{i}\right)_{i \in[r]_{0}},\left(V_{i}\right)_{i \in[r]_{0}}\right)$ is an $(\varepsilon, d)$-super-regular blow-up instance with exceptional sets $\left(X_{0}, V_{0}\right)$ if $X_{0}$ is an independent set in $H,\left|V_{0}\right|=\left|X_{0}\right|$ and $\left(H-X_{0}, G-V_{0},\left(X_{i}\right)_{i \in[r]},\left(V_{i}\right)_{i \in[r]}\right)$ is an $(\varepsilon, d)$-super-regular blow-up instance. We call graphs $\left(A_{i}\right)_{i \in[r]}$ candidacy graphs if $A_{i}$ is a bipartite graph with partition $\left(X_{i}, V_{i}\right)$ for all $i \in[r]$.

Lemma 4.7 ([17, Lemma 5.2]). Suppose $1 / n \ll \varepsilon, \mu \ll d, 1 / r, 1 / \Delta$. Let $\mathcal{B}=\left(H, G,\left(X_{i}\right)_{i \in[r]_{0}}\right.$, $\left.\left(V_{i}\right)_{i \in[r]_{0}}\right)$ be an $\left(\varepsilon, d_{G}\right)$-super-regular blow-up instance with exceptional sets $\left(X_{0}, V_{0}\right)$ and $\left(\varepsilon, d_{A}\right)$ super-regular candidacy graphs $\left(A_{i}\right)_{i \in[r]}$, where $d_{G}, d_{A} \geq d$. Assume further that 
(i) $\Delta(H) \leq \Delta$.

(ii) $\left|V_{i}\right|=n$ for all $i \in[r]$.

(iii) $H\left[X_{i}, X_{j}\right]$ is a matching for all $i j \in\left(\begin{array}{c}{[r]} \\ 2\end{array}\right)$.

Let $c: E(G) \rightarrow C$ be a $\mu$-bounded edge-colouring of $G$. Suppose a bijection $\psi_{0}: X_{0} \rightarrow V_{0}$ is given such that

(iv) For all $x \in X_{0}, i \in[r]$ and $x_{i} \in N_{H}(x) \cap X_{i}$, we have $N_{A_{i}}\left(x_{i}\right) \subseteq N_{G}\left(\psi_{0}(x)\right)$.

(v) For all $i \in[r], x \in X_{i}, v \in N_{A_{i}}(x)$ and distinct $x_{0}, x_{0}^{\prime} \in N_{H}(x) \cap X_{0}$, we have $c\left(\psi_{0}\left(x_{0}\right) v\right) \neq$ $c\left(\psi_{0}\left(x_{0}^{\prime}\right) v\right)$.

Then there exists a rainbow embedding $\psi$ of $H$ into $G$ that extends $\psi_{0}$ such that $\psi(x) \in N_{A_{i}}(x)$ for all $i \in[r]$ and $x \in X_{i}$.

\section{Colour splitting}

The goal of this section is to provide some useful lemmas to refine the partitions of a blow-up instance and split the colours into groups to obtain better control for the rainbow embedding. In particular, we will refine the partition of $H$ using the Hajnal-Szemerédi theorem (Theorem 5.3), and we will refine the partition of $G$ accordingly by a random procedure. This reduction is performed in Lemma 5.4. Additionally, we group the edges of $G$ such that the edge-colouring of $G$ is colour-split, which is based on a random procedure given in Lemma 5.1. To obtain better control on the boundedness condition of the edge-colouring of a blow-up instance when performing these reductions, we first group the edges of $G$ such that $G$ is colour-split (Lemma 5.2) and afterward refine the partitions of $H$ and $G$ (Lemma 5.4).

The first lemma will guarantee that with high probability, the resulting graph is still super-regular when we randomly split colours to obtain a colour-split colouring.

Lemma 5.1. Let $1 / n \ll \varepsilon \ll \varepsilon^{\prime} \ll \gamma, d, 1 / \Lambda$. Suppose $G$ is an $(\varepsilon, d)$-super-regular graph with vertex partition $(A, B)$ such that $|A|,|B|=(1 \pm \varepsilon) n$, and $c: E(G) \rightarrow C$ is a locally $\Lambda$-bounded edge-colouring of $G$. Suppose $\left\{Y_{\alpha}: \alpha \in C\right\} \cup\left\{Z_{e}: e \in E(G)\right\}$ is a set of mutually independent Bernoulli random variables such that $\mathbb{P}\left[Y_{c(e)}+Z_{e}=2\right]=\gamma$ for every $e \in E(G)$. Suppose $G^{\prime}$ is the random spanning subgraph of $G$ where $e \in E(G)$ belongs to $E\left(G^{\prime}\right)$ whenever $Y_{c(e)}+Z_{e}=2$. Then $G^{\prime}$ is $\left(\varepsilon^{\prime}, \gamma d\right)$-superregular with probability at least $1-1 / n^{10}$.

Proof. We call a pair of distinct vertices $u, v \in A \operatorname{good}$ if $\left|N_{G}(u, v)\right|=(d \pm \varepsilon)^{2}|B|$ and $\mid\{w \in$ $\left.N_{G}(u, v): c(u w)=c(v w)\right\}|\leq \varepsilon| B \mid$. We first claim that almost all pairs are good.

Claim 1. There are at least $(1-7 \varepsilon)|A|^{2} / 2 \operatorname{good}$ pairs $u, v \in A$.

Proof of claim: Since $G$ is $(\varepsilon, d)$-super-regular, at most $2 \varepsilon|A|^{2}$ pairs $u, v \in A$ do not satisfy $\left|N_{G}(u, v)\right|=$ $(d \pm \varepsilon)^{2}|B|$ by Fact 4.4 .

We claim that the number of pairs $u, v \in A$ with $\left|\left\{w \in N_{G}(u, v): c(u w)=c(v w)\right\}\right| \geq \varepsilon|B|$ is at most $\varepsilon|A|^{2}$. For this, we first count the number of monochromatic paths of length 2 in $G$ with both ends in $A$. Each vertex $w \in B$ is contained in $\sum_{\alpha \in C}\left({ }_{\operatorname{deg}_{G}^{\alpha}(w)}^{\alpha}\right)$ monochromatic paths $u w v$ in $G$. Since $\operatorname{deg}_{G}^{\alpha}(w) \leq \Lambda$ for every colour $\alpha \in C$ and $\sum_{\alpha \in C} \operatorname{deg}_{G}^{\alpha}(w)^{2} \leq|A|$, we have

$$
\sum_{\alpha \in C}\left(\begin{array}{c}
\operatorname{deg}_{G}^{\alpha}(w) \\
2
\end{array}\right) \leq \sum_{\alpha \in C} \operatorname{deg}_{G}^{\alpha}(w)^{2} \leq \Lambda|A| .
$$

Hence, there are at most $\Lambda|A||B|$ monochromatic paths of length 2 in $G$ with both ends in $A$. This implies that the number of pairs $u, v \in A$ with $\left|\left\{w \in N_{G}(u, v): c(u w)=c(v w)\right\}\right| \geq \varepsilon|B|$ is at most

$$
\frac{\Lambda|A||B|}{\varepsilon|B|} \leq \varepsilon|A|^{2}
$$


Thus, there are at least $\left(\begin{array}{c}|A| \\ 2\end{array}\right)-3 \varepsilon|A|^{2} \geq(1-7 \varepsilon)|A|^{2} / 2$ good pairs $u, v \in A$.

We fix a vertex $x \in A \cup B$ and a good pair of vertices $u, v \in A$. Let $X_{x}:=\operatorname{deg}_{G^{\prime}}(x)$ and $X_{u, v}:=$ $\left|N_{G^{\prime}}(u, v)\right|$. Clearly, $X_{x}$ and $X_{u, v}$ are determined by $\left\{Y_{\alpha}: \alpha \in C\right\} \cup\left\{Z_{e}: e \in E(G)\right\}$. Note that if $w \in N_{G}(u, v)$ satisfies $c(u w) \neq c(v w)$, then $\mathbb{P}\left[w \in N_{G^{\prime}}(u, v)\right]=\gamma^{2}$. Thus, we have

$$
\mathbb{E}\left[X_{x}\right]=\gamma \operatorname{deg}_{G}(x)=\gamma d n \pm 3 \varepsilon n \text { and } \mathbb{E}\left[X_{u, v}\right]=\gamma^{2} d^{2} n \pm 10 \varepsilon n .
$$

For all $\alpha \in C$ and $e \in E(G)$, let $b_{\alpha}$ and $b_{e}$ be minimally chosen such that changing the outcome of $Y_{\alpha}$ changes $X_{x}$ by at most $b_{\alpha}$, and changing the outcome of $Z_{e}$ changes $X_{x}$ by at most $b_{e}$. Note that

$$
\sum_{\alpha \in C} b_{\alpha}+\sum_{e \in E(G)} b_{e} \leq 2 \operatorname{deg}_{G}(x) \leq 3 n .
$$

Moreover, we clearly have $b_{e} \leq 1$, and since the colouring $c$ is locally $\Lambda$-bounded, $b_{\alpha} \leq \Lambda$. Using McDiarmid's inequality (Theorem 4.2), we obtain that

$$
\mathbb{P}\left[\left|X_{x}-\mathbb{E}\left[X_{x}\right]\right|>\varepsilon n\right] \leq 2 \exp \left(-\frac{\varepsilon^{2} n^{2}}{\Lambda \cdot 3 n}\right)<\frac{1}{n^{20}} .
$$

With similar arguments, one can show that

$$
\mathbb{P}\left[\left|X_{u, v}-\mathbb{E}\left[X_{u, v}\right]\right|>\varepsilon n\right]<\frac{1}{n^{20}} .
$$

A union bound over all $x \in A \cup B$ and all good pairs $u, v \in A$ yields together with (5.1), (5.2), and (5.3) that with probability at least $1-1 / n^{10}$, we have $\operatorname{deg}_{G^{\prime}}(x)=\gamma d n \pm 4 \varepsilon n$ for all $x \in A \cup B$, and $\left|N_{G^{\prime}}(u, v)\right|=\gamma^{2} d^{2} n \pm 11 \varepsilon n$ for all good pairs $u, v \in A$. Given that, Theorem 4.6 implies that $G^{\prime}$ is $\left(\varepsilon^{\prime}, \gamma d\right)$-super-regular.

The next lemma states that we can split the colours of the host graph $G$ into groups and obtain a subgraph $G^{\prime}$ that is still super-regular, and whose colouring is colour-split and appropriately bounded.

Lemma 5.2. Let $1 / n \ll \varepsilon \ll \varepsilon^{\prime} \ll d^{\prime} \ll \gamma \ll d, 1 / \Lambda, 1 / r, 1 / \Delta$. Suppose $\left(H, G,\left(X_{i}\right)_{i \in[r]},\left(V_{i}\right)_{i \in[r]}\right)$ is an $(\varepsilon, d)$-super-regular blow-up instance. Assume further that

(i) $\Delta(H) \leq \Delta$ and $e_{H}\left(X_{i}, X_{j}\right) \geq \gamma^{2} n$ for all $i j \in\left(\begin{array}{c}{[r]} \\ 2\end{array}\right)$.

(ii) $\left|V_{i}\right|=(1 \pm \varepsilon) n$ for all $i \in[r]$.

(iii) $c: E(G) \rightarrow C$ is locally $\Lambda$-bounded and the following holds for all $\alpha \in C$ :

$$
\sum_{i j \in\left(\begin{array}{c}
{[r]} \\
2
\end{array}\right)} e_{G}^{\alpha}\left(V_{i}, V_{j}\right) e_{H}\left(X_{i}, X_{j}\right) \leq(1-\gamma) d n^{2} .
$$

Then there exists a spanning subgraph $G^{\prime}$ of $G$ such that

(a) $\left(H, G^{\prime},\left(X_{i}\right)_{i \in[r]},\left(V_{i}\right)_{i \in[r]}\right)$ is an $\left(\varepsilon^{\prime}, d^{\prime}\right)$-super-regular blow-up instance.

(b) c restricted to $G^{\prime}$ is colour-split.

(c) c restricted to $G^{\prime}\left[V_{i}, V_{j}\right]$ is $\left(1-\frac{\gamma}{2}\right) \frac{e_{G^{\prime}}\left(V_{i}, V_{j}\right)}{e_{H}\left(X_{i}, X_{j}\right)}$-bounded for all $i j \in\left(\begin{array}{c}{[r]} \\ 2\end{array}\right)$.

Proof. Let $\hat{\varepsilon}$ be such that $\varepsilon \ll \hat{\varepsilon} \ll \varepsilon^{\prime}$. The proof proceeds in three steps, where we iteratively define spanning subgraphs $G_{3} \subseteq G_{2} \subseteq G_{1} \subseteq G$ such that $G_{3}$ satisfies the required properties of $G^{\prime}$ in the statement.

In the first step, we suitably sparsify each bipartite subgraph $G\left[V_{i}, V_{j}\right]$. For every $i j \in\left(\begin{array}{c}{[r]} \\ 2\end{array}\right)$, let

$$
p_{i j}:=\frac{e_{H}\left(X_{i}, X_{j}\right)}{2 \Delta n} .
$$


Note that $\gamma^{2} /(2 \Delta) \leq p_{i j} \leq 1$ since $\gamma^{2} n \leq e_{H}\left(X_{i}, X_{j}\right) \leq \Delta\left|X_{i}\right| \leq 2 \Delta n$. For every $i j \in\left(\begin{array}{c}{[r]} \\ 2\end{array}\right)$, we keep each edge of $G\left[V_{i}, V_{j}\right]$ independently at random with probability $p_{i j}$ and denote the resulting graph by $G_{1}\left[V_{i}, V_{j}\right]$. A simple application of Chernoff's inequality together with a union bound yields the following claim.

Claim 1. The following properties hold simultaneously with probability at least $1-1 / n$ for every $i j \in\left(\begin{array}{c}{[r]} \\ 2\end{array}\right)$.

(C1.1) $G_{1}\left[V_{i}, V_{j}\right]$ is $\left(2 \varepsilon, p_{i j} d\right)$-super-regular;

(C1.2) $e_{G_{1}}^{\alpha}\left(V_{i}, V_{j}\right) \leq e_{G}^{\alpha}\left(V_{i}, V_{j}\right) p_{i j}+\varepsilon n$ for every colour $\alpha \in C$.

Hence, by Claim 1, we may assume that $G_{1}$ is a spanning subgraph of $G$ such that properties (C1.1)(C1.2) hold. For every colour $\alpha \in C$, we obtain that

$$
\begin{aligned}
& \sum_{i j \in\left(\begin{array}{c}
{[r]} \\
2
\end{array}\right)} e_{G_{1}}^{\alpha}\left(V_{i}, V_{j}\right) \frac{e_{H}\left(X_{i}, X_{j}\right)}{e_{G_{1}}\left(V_{i}, V_{j}\right)} \stackrel{(\mathrm{C} 1.1),(\mathrm{C} 1.2)}{\leq} \sum_{i j \in\left(\begin{array}{c}
{[r]} \\
2
\end{array}\right)}\left(e_{G}^{\alpha}\left(V_{i}, V_{j}\right) p_{i j}+\varepsilon n\right) \frac{e_{H}\left(X_{i}, X_{j}\right)}{\left(1-\varepsilon^{1 / 2}\right) p_{i j} d n^{2}} \\
& \leq\left(1+2 \varepsilon^{1 / 2}\right) \sum_{i j \in\left(\begin{array}{c}
{[r]} \\
2
\end{array}\right)} e_{G}^{\alpha}\left(V_{i}, V_{j}\right) \frac{e_{H}\left(X_{i}, X_{j}\right)}{d n^{2}}+\varepsilon n \sum_{i j \in\left(\begin{array}{c}
{[r]} \\
2
\end{array}\right)} \frac{2 e_{H}\left(X_{i}, X_{j}\right)}{p_{i j} d n^{2}} \\
& \stackrel{(\text { iii),(5.4) }}{\leq}\left(1+2 \varepsilon^{1 / 2}\right)(1-\gamma)+\varepsilon n\left(\begin{array}{c}
r \\
2
\end{array}\right) \frac{4 \Delta n}{d n^{2}} \leq 1-\gamma+3 \varepsilon^{1 / 2} \leq 1-\frac{3 \gamma}{4} .
\end{aligned}
$$

Note that (5.4) and (C1.1) imply that

$$
\frac{e_{G_{1}}\left(V_{i}, V_{j}\right)}{e_{H}\left(X_{i}, X_{j}\right)}=\frac{\left(d \pm \varepsilon^{1 / 2}\right) n}{2 \Delta}
$$

Hence, for every colour $\alpha \in C$, we obtain

$$
\begin{gathered}
e^{\alpha}\left(G_{1}\right)=\sum_{i j \in\left(\begin{array}{c}
{[r]} \\
2
\end{array}\right)} e_{G_{1}}^{\alpha}\left(V_{i}, V_{j}\right) \stackrel{(5.6)}{\leq} \frac{\left(d+\varepsilon^{1 / 2}\right) n}{2 \Delta} \sum_{i j \in\left(\begin{array}{c}
{[r]} \\
2
\end{array}\right)} e_{G_{1}}^{\alpha}\left(V_{i}, V_{j}\right) \frac{e_{H}\left(X_{i}, X_{j}\right)}{e_{G_{1}}\left(V_{i}, V_{j}\right)} \\
\stackrel{(5.5)}{\leq}\left(1-\frac{3 \gamma}{4}\right) \frac{\left(d+\varepsilon^{1 / 2}\right) n}{2 \Delta} .
\end{gathered}
$$

In the next step, we define a random subgraph $G_{2} \subseteq G_{1}$. This will ensure that the final colouring is colour-split. We choose $\tau: C \rightarrow\left(\begin{array}{c}{[r]} \\ 2\end{array}\right)$, where each $\tau(\alpha)$ is chosen independently at random according to some probability distribution $\left(q_{i j}^{\alpha}\right)_{i j \in\left(\begin{array}{c}{[r]} \\ 2\end{array}\right)}$; and for each $i j \in\left(\begin{array}{c}{[r]} \\ 2\end{array}\right)$ and each edge $e$ of $G_{1}\left[V_{i}, V_{j}\right]$, let $Z_{e}$ be a Bernoulli random variable with parameter $\gamma^{2} / q_{i j}^{c(e)}$, all independent and independent of the choice of $\tau$. Define $G_{2}$ by keeping each edge $e \in E_{G_{1}}\left(V_{i}, V_{j}\right)$ if $\tau(c(e))=i j$ and $Z_{e}=1$. Hence,

$$
\text { for all } e \in E\left(G_{1}\right) \text {, we have } \mathbb{P}\left[e \in E\left(G_{2}\right)\right]=\gamma^{2} \text {. }
$$

We define $q_{i j}^{\alpha}$ as follows. For all $\alpha \in C$, let

$$
\mathcal{J}^{\alpha}:=\left\{i j \in\left(\begin{array}{c}
{[r]} \\
2
\end{array}\right): e_{G_{1}}^{\alpha}\left(V_{i}, V_{j}\right)>\frac{\gamma^{2} e^{\alpha}\left(G_{1}\right)}{1-\left(\begin{array}{c}
r \\
2
\end{array}\right) \gamma^{2}}\right\} \quad \text { and } \quad \overline{J^{\alpha}}:=\left(\begin{array}{c}
{[r]} \\
2
\end{array}\right) \backslash \mathcal{J}^{\alpha} \text {. }
$$


For $i j \in \overline{J^{\alpha}}$, we set $q_{i j}^{\alpha}:=\gamma^{2}$. For $i j \in \mathcal{J}^{\alpha}$, we set

$$
q_{i j}^{\alpha}:=\left(1-\left|\overline{J^{\alpha}}\right| \gamma^{2}\right) \frac{e_{G_{1}}^{\alpha}\left(V_{i}, V_{j}\right)}{\sum_{i^{\prime} j^{\prime} \in \mathcal{J}^{\alpha}} e_{G_{1}}^{\alpha}\left(V_{i^{\prime}}, V_{j^{\prime}}\right)} .
$$

Note that $\gamma^{2} \leq q_{i j}^{\alpha} \leq 1$ for all $i j \in\left(\begin{array}{c}{[r]} \\ 2\end{array}\right)$, and $\sum_{i j \in\left(\begin{array}{c}{[r]} \\ 2\end{array}\right)} q_{i j}^{\alpha}=1$.

Claim 2. The following properties hold simultaneously with probability at least $1-1 / n$ for every $i j \in\left(\begin{array}{c}{[r]} \\ 2\end{array}\right)$ and every colour $\alpha \in C$.

(C2.1) $G_{2}\left[V_{i}, V_{j}\right]$ is $\left(\hat{\varepsilon}, \gamma^{2} p_{i j} d\right)$-super-regular.

(C2.2) $e_{G_{2}}^{\alpha}\left(V_{i}, V_{j}\right) \leq \frac{\gamma^{2}}{q_{i j}^{\alpha}} e_{G_{1}}^{\alpha}\left(V_{i}, V_{j}\right)+\varepsilon n$.

Proof of claim: For every $i j \in\left(\begin{array}{c}{[r]} \\ 2\end{array}\right)$, by (5.8) and (C1.1), Lemma 5.1 with $Y_{\alpha}=\mathbb{1}_{\tau(\alpha)=i j}$ and $Z_{e}$ as defined above implies that $(\mathrm{C} 2.1)$ holds with probability at least $1-1 / n^{5}$.

To verify (C2.2), note that for $i j \in\left(\begin{array}{c}{[r]} \\ 2\end{array}\right)$, the colour $\alpha$ appears in $G_{2}\left[V_{i}, V_{j}\right]$ only if $\tau(\alpha)=i j$. Since we keep each $\alpha$-coloured edge independently at random with probability $\gamma^{2} / q_{i j}^{\alpha}$, a simple application of Chernoff's inequality yields that (C2.2) holds with probability at least $1-1 / n^{5}$.

Hence, by Claim 2, we may assume that $G_{2}$ is a spanning subgraph of $G_{1}$ such that properties (C2.1) and (C2.2) hold. By the construction of $G_{2}$, the restricted colouring $\left.c\right|_{E\left(G_{2}\right)}$ is colour-split.

We also show that the required boundedness condition is satisfied; see (5.14) below. For $i j \in\left(\begin{array}{c}{[r]} \\ 2\end{array}\right)$, we deduce from (5.4) and (C2.1) that

$$
e_{G_{2}}\left(V_{i}, V_{j}\right)=\gamma^{2} p_{i j}\left(d \pm \hat{\varepsilon}^{1 / 2}\right) n^{2} \stackrel{(5.4)}{=}\left(d \pm \hat{\varepsilon}^{1 / 2}\right) \frac{\gamma^{2} n}{2 \Delta} e_{H}\left(X_{i}, X_{j}\right) .
$$

For a colour $\alpha \in C$ and $i j \in \overline{J^{\alpha}}$, as $G_{2} \subseteq G_{1}$, we obtain that

$$
e_{G_{2}}^{\alpha}\left(V_{i}, V_{j}\right) \leq e_{G_{1}}^{\alpha}\left(V_{i}, V_{j}\right) \stackrel{(5.9)}{\leq} \frac{\gamma^{2}}{1-\left(\begin{array}{l}
r \\
2
\end{array}\right) \gamma^{2}} e^{\alpha}\left(G_{1}\right) .
$$

For a colour $\alpha \in C$ and $i j \in \mathcal{J}^{\alpha}$, we obtain with (C2.2) that

$$
\begin{aligned}
& e_{G_{2}}^{\alpha}\left(V_{i}, V_{j}\right) \stackrel{(5.10)}{\leq} \frac{\gamma^{2}}{1-\left|\overline{J^{\alpha}}\right| \gamma^{2}} \cdot e_{G_{1}}^{\alpha}\left(V_{i}, V_{j}\right) \frac{\sum_{i^{\prime} j^{\prime} \in \mathcal{J}^{\alpha}} e_{G_{1}}^{\alpha}\left(V_{i^{\prime}}, V_{j^{\prime}}\right)}{e_{G_{1}}^{\alpha}\left(V_{i}, V_{j}\right)}+\varepsilon n \\
& \leq \frac{\gamma^{2}}{1-\left(\begin{array}{c}
r \\
2
\end{array}\right) \gamma^{2}} \sum_{i^{\prime} j^{\prime} \in\left(\begin{array}{c}
{[r]} \\
2
\end{array}\right)} e_{G_{1}}^{\alpha}\left(V_{i^{\prime}}, V_{j^{\prime}}\right)+\varepsilon n=\frac{\gamma^{2}}{1-\left(\begin{array}{c}
r \\
2
\end{array}\right) \gamma^{2}} e^{\alpha}\left(G_{1}\right)+\varepsilon n .
\end{aligned}
$$

Moreover, for every colour $\alpha \in C$ and every $i j \in\left(\begin{array}{c}{[r]} \\ 2\end{array}\right)$, we conclude that

$$
\begin{gathered}
\frac{\gamma^{2}}{1-\left(\begin{array}{c}
r \\
2
\end{array}\right) \gamma^{2}} e^{\alpha}\left(G_{1}\right)+\varepsilon n \stackrel{(5.7)}{\leq} \frac{1-3 \gamma / 4}{1-\left(\begin{array}{c}
r \\
2
\end{array}\right) \gamma^{2}} \cdot \frac{\gamma^{2} n}{2 \Delta}\left(d+\varepsilon^{1 / 2}\right)+\varepsilon n \\
\stackrel{\text { (5.11) }}{\leq} \frac{1-3 \gamma / 4}{1-\left(\begin{array}{c}
r \\
2
\end{array}\right) \gamma^{2}} \cdot \frac{e_{G_{2}}\left(V_{i}, V_{j}\right)}{e_{H}\left(X_{i}, X_{j}\right)} \cdot \frac{d+\varepsilon^{1 / 2}}{d-\hat{\varepsilon}^{1 / 2}}+\varepsilon n \leq\left(1-\frac{2 \gamma}{3}\right) \frac{e_{G_{2}}\left(V_{i}, V_{j}\right)}{e_{H}\left(X_{i}, X_{j}\right)},
\end{gathered}
$$

which implies together with (5.12) and (5.13) that for every colour $\alpha \in C$ and every $i j \in\left(\begin{array}{c}{[r]} \\ 2\end{array}\right)$,

$$
e_{G_{2}}^{\alpha}\left(V_{i}, V_{j}\right) \leq\left(1-\frac{2 \gamma}{3}\right) \frac{e_{G_{2}}\left(V_{i}, V_{j}\right)}{e_{H}\left(X_{i}, X_{j}\right)} .
$$


Let $G_{3}$ be a spanning subgraph of $G_{2}$ where, for each bipartite pair $G_{2}\left[V_{i}, V_{j}\right]$, we keep each edge independently at random with probability $d^{\prime} /\left(\gamma^{2} p_{i j} d\right)$. As $G_{2}\left[V_{i}, V_{j}\right]$ is $\left(\hat{\varepsilon}, \gamma^{2} p_{i j} d\right)$-super-regular, we may conclude by simple applications of Chernoff's inequality that with probability at least $1-1 / n$ for all $i j \in\left(\begin{array}{c}{[r]} \\ 2\end{array}\right)$, the graph $G_{3}\left[V_{i}, V_{j}\right]$ is $\left(\varepsilon^{\prime}, d^{\prime}\right)$-super-regular, and for every colour $\alpha \in C$, we have

$$
e_{G_{3}}^{\alpha}\left(V_{i}, V_{j}\right) \leq\left(1-\frac{\gamma}{2}\right) \frac{e_{G_{3}}\left(V_{i}, V_{j}\right)}{e_{H}\left(X_{i}, X_{j}\right)}
$$

due to (5.14). Clearly, also $c$ restricted to $G_{3}$ is colour-split. Hence, we conclude that there is a spanning subgraph $G_{3}$ of $G_{2}$ satisfying properties (a)-(c), which implies the statement with $G_{3}$ playing the role of $G^{\prime}$.

The next lemma states that we can refine the partitions of a blow-up instance $\left(H, G,\left(X_{i}\right)_{i \in[r]}\right.$, $\left.\left(V_{i}\right)_{i \in[r]}\right)$ where the edge-colouring of $G$ is colour-split such that $H$ only induces matchings between its refined partition classes, and the bipartite pairs of $G$ are still super-regular and colour-split. Similar as in the reduction in [42], we first apply the Hajnal-Szemerédi theorem to $H^{2}\left[X_{i}\right]$ for each cluster $X_{i}$ to obtain a refined partition of $H$ where every cluster is now 2-independent. Accordingly, we refine the partition of $G$ randomly to preserve the super-regularity. Additionally, we partition the colours into disjoint colour sets such that the colouring between the refined partitions of $G$ is still colour-split.

We first state the classical Hajnal-Szemerédi theorem.

Theorem 5.3 ([22]). Let $G$ be a graph on $n$ vertices with $\Delta(G)<k \leq n$. Then $V(G)$ can be partitioned into $k$ independent sets of size $\left\lfloor\frac{n}{k}\right\rfloor$ or $\left\lceil\frac{n}{k}\right\rceil$.

Lemma 5.4. Let $1 / n \ll \varepsilon \ll \varepsilon^{\prime} \ll d^{\prime} \ll \gamma \ll d, 1 / \Lambda, 1 / r, 1 / \Delta$. Suppose $\left(H, G,\left(X_{i}\right)_{i \in[r]},\left(V_{i}\right)_{i \in[r]}\right)$ is an $(\varepsilon, d)$-super-regular blow-up instance. Assume further that

(i) $\Delta(H) \leq \Delta$ and $e_{H}\left(X_{i}, X_{j}\right) \geq \gamma^{2} n$ for all $i j \in\left(\begin{array}{c}{[r]} \\ 2\end{array}\right)$.

(ii) $\left|V_{i}\right|=(1 \pm \varepsilon) n$ for all $i \in[r]$.

(iii) $c: E(G) \rightarrow C$ is a colour-split edge-colouring such that $c$ is locally $\Lambda$-bounded and $c$ restricted to $G\left[V_{i}, V_{j}\right]$ is $(1-\gamma) e_{G}\left(V_{i}, V_{j}\right) / e_{H}\left(X_{i}, X_{j}\right)$-bounded for all $i j \in\left(\begin{array}{c}{[r]} \\ 2\end{array}\right)$.

Then there exists an $\left(\varepsilon^{\prime}, d^{\prime}\right)$-super-regular blow-up instance $\left(H^{\prime}, G^{\prime},\left(X_{i, j}\right)_{i \in[r], j \in\left[\Delta^{2}\right]},\left(V_{i, j}\right)_{i \in[r], j \in\left[\Delta^{2}\right]}\right)$ such that

(a) $\left(X_{i, j}\right)_{j \in\left[\Delta^{2}\right]}$ is partition of $X_{i}$ and $\left(V_{i, j}\right)_{j \in\left[\Delta^{2}\right]}$ is partition of $V_{i}$ for every $i \in[r]$, and $\left|X_{i, j}\right|=$ $\left|V_{i, j}\right|=\left(1 \pm \varepsilon^{\prime}\right) n / \Delta^{2}$ for all $i \in[r], j \in\left[\Delta^{2}\right]$.

(b) $H^{\prime}$ is a supergraph of $H$ on $V(H)$ such that $H^{\prime}\left[X_{i_{1}, j_{1}}, X_{i_{2}, j_{2}}\right]$ is a matching of size at least $\gamma^{4} n / \Delta^{2}$ for all $i_{1}, i_{2} \in[r], j_{1}, j_{2} \in\left[\Delta^{2}\right],\left(i_{1}, j_{1}\right) \neq\left(i_{2}, j_{2}\right)$.

(c) $G^{\prime}$ is a graph on $V(G)$ such that $G^{\prime}\left[V_{i_{1}, j_{1}}, V_{i_{2}, j_{2}}\right] \subseteq G\left[V_{i_{1}}, V_{i_{2}}\right]$ for all distinct $i_{1}, i_{2} \in[r]$ and all $j_{1}, j_{2} \in\left[\Delta^{2}\right]$.

(d) $c^{\prime}: E\left(G^{\prime}\right) \rightarrow C^{\prime}$ is an edge-colouring of $G^{\prime}$ such that $\left.c^{\prime}\right|_{E(G) \cap E\left(G^{\prime}\right)}=\left.c\right|_{E(G) \cap E\left(G^{\prime}\right)}$, and $c^{\prime}$ is colour-split with respect to the partition $\left(V_{i, j}\right)_{i \in[r], j \in\left[\Delta^{2}\right]}$, and $c^{\prime}$ is locally $\Lambda$-bounded, and $c^{\prime}$ restricted to $G^{\prime}\left[V_{i_{1}, j_{1}}, V_{i_{2}, j_{2}}\right]$ is

$$
\left(1-\frac{\gamma}{2}\right) \frac{e_{G^{\prime}}\left(V_{i_{1}, j_{1}}, V_{i_{2}, j_{2}}\right)}{e_{H^{\prime}}\left(X_{i_{1}, j_{1}}, X_{i_{2}, j_{2}}\right)} \text {-bounded }
$$

for all $i_{1}, i_{2} \in[r], j_{1}, j_{2} \in\left[\Delta^{2}\right],\left(i_{1}, j_{1}\right) \neq\left(i_{2}, j_{2}\right)$.

Proof. Since $c: E(G) \rightarrow C$ is colour-split, we may assume that $c$ is the union of edge-colourings $c_{i_{1} i_{2}}: E\left(G\left[V_{i_{1}}, V_{i_{2}}\right]\right) \rightarrow C_{i_{1} i_{2}}$ for $i_{1} i_{2} \in\left(\begin{array}{c}{[r]} \\ 2\end{array}\right)$, where $C_{i_{1} i_{2}} \cap C_{i_{1}^{\prime} i_{2}^{\prime}}=\emptyset$ for distinct $i_{1} i_{2}, i_{1}^{\prime} i_{2}^{\prime} \in\left(\begin{array}{c}{[r]} \\ 2\end{array}\right)$.

First, we apply Theorem 5.3 to $H^{2}\left[X_{i}\right]$ for every $i \in[r]$. Since $\Delta\left(H^{2}\left[X_{i}\right]\right) \leq \Delta^{2}-1$, there exists a partition of $X_{i}$ into 2-independent sets $X_{i, 1}, \ldots, X_{i, \Delta^{2}}$ in $H$, each of size $\left|X_{i}\right| / \Delta^{2} \pm 1=(1 \pm 2 \varepsilon) n^{\prime}$, where 
$n^{\prime}:=n / \Delta^{2}$. Hence for all $i_{1}, i_{2} \in[r], j_{1}, j_{2} \in\left[\Delta^{2}\right]$, the bipartite graph $H\left[X_{i_{1}, j_{1}}, X_{i_{2}, j_{2}}\right]$ is a (possibly empty) matching. Clearly, we can add a minimal number of edges to $H$ to obtain a supergraph $H^{\prime}$ such that $H^{\prime}\left[X_{i_{1}, j_{1}}, X_{i_{2}, j_{2}}\right]$ is a matching of size at least $\gamma^{4} n^{\prime}$ for all $i_{1}, i_{2} \in[r], j_{1}, j_{2} \in\left[\Delta^{2}\right],\left(i_{1}, j_{1}\right) \neq\left(i_{2}, j_{2}\right)$, which yields (b).

To obtain (a), we refine the partition of $V(G)$ accordingly. We claim that the following partitions exist. For every $i \in[r]$, let $\left(V_{i, j}\right)_{j \in\left[\Delta^{2}\right]}$ be a partition of $V_{i}$ such that $\left|V_{i, j}\right|=\left|X_{i, j}\right|$ for every $j \in\left[\Delta^{2}\right]$, and such that for all distinct $i_{1}, i_{2} \in[r]$, all $j_{1}, j_{2} \in\left[\Delta^{2}\right]$, and $v \in V_{i_{1}, j_{1}} \cup V_{i_{2}, j_{2}}$, we have

$$
\operatorname{deg}_{G\left[V_{i_{1}, j_{1}}, V_{i_{2}, j_{2}}\right]}(v)=(d \pm 3 \varepsilon) n^{\prime}
$$

and

$$
\left.c\right|_{E\left(G\left[V_{i_{1}, j_{1}}, V_{i_{2}, j_{2}}\right]\right)} \text { is }(1-\gamma+\varepsilon) \frac{(d+3 \varepsilon) n^{\prime 2}}{e_{H}\left(X_{i_{1}}, X_{i_{2}}\right)} \text {-bounded. }
$$

That such a partition exists can be seen by a probabilistic argument as follows: For each $i \in[r]$, let $\tau_{i}: V_{i} \rightarrow\left[\Delta^{2}\right]$, where $\tau_{i}(v)$ is chosen uniformly at random for every $v \in V_{i}$, all independently, and let $V_{i, j}:=\left\{v \in V_{i}: \tau_{i}(v)=j\right\}$ for every $j \in\left[\Delta^{2}\right]$. McDiarmid's inequality together with a union bound implies that (5.15) and (5.16) hold with probability at least $1-\mathrm{e}^{-\sqrt{n}}$. Moreover, standard properties of the multinomial distribution yield that $\left|V_{i, j}\right|=\left|X_{i, j}\right|$ for all $i \in[r], j \in\left[\Delta^{2}\right]$ with probability at least $\Omega\left(n^{-\Delta^{2} r}\right)$.

Thus, for every $i \in[r]$, there exists a partition $\left(V_{i, j}\right)_{j \in\left[\Delta^{2}\right]}$ of $V_{i}$ with the required properties.

Since $G\left[V_{i_{1}}, V_{i_{2}}\right]$ is $(\varepsilon, d)$-super-regular and due to (5.15), it follows that for all distinct $i_{1}, i_{2} \in[r]$ and all $j_{1}, j_{2} \in\left[\Delta^{2}\right]$, the graph $G\left[V_{i_{1}, j_{1}}, V_{i_{2}, j_{2}}\right]$ is $\left(2 \Delta^{2} \varepsilon, d\right)$-super-regular. By the construction of the supergraph $H^{\prime}$, we have added at most $\gamma^{4} \Delta^{2} n$ edges to each pair $\left(X_{i_{1}}, X_{i_{2}}\right)$ in $H$. Hence for all distinct $i_{1}, i_{2} \in[r]$,

$$
e_{H}\left(X_{i_{1}}, X_{i_{2}}\right) \geq e_{H^{\prime}}\left(X_{i_{1}}, X_{i_{2}}\right)-\gamma^{4} \Delta^{2} n \geq e_{H^{\prime}}\left(X_{i_{1}}, X_{i_{2}}\right)\left(1-\gamma^{2} \Delta^{2}\right),
$$

where the last inequality holds since $e_{H^{\prime}}\left(X_{i_{1}}, X_{i_{2}}\right) \geq e_{H}\left(X_{i_{1}}, X_{i_{2}}\right) \geq \gamma^{2} n$. Now (5.16) and (5.17) imply that for all distinct $i_{1}, i_{2} \in[r]$ and all $j_{1}, j_{2} \in\left[\Delta^{2}\right]$, the colouring

$$
\left.c\right|_{E\left(G\left[V_{i_{1}, j_{1}}, V_{i_{2}, j_{2}}\right]\right)} \text { is }\left(1-\frac{3 \gamma}{4}\right) \frac{(d+3 \varepsilon) n^{\prime 2}}{e_{H^{\prime}}\left(X_{i_{1}}, X_{i_{2}}\right)} \text {-bounded. }
$$

Next, we iteratively define spanning subgraphs $G_{2} \subseteq G_{1} \subseteq G$ and a supergraph $G^{\prime} \supseteq G_{2}$ that satisfies the required properties in the statement.

First, we claim that there exists a spanning subgraph $G_{1} \subseteq G$ that is colour-split with respect to the partition $\left(V_{i, j}\right)_{i \in[r], j \in\left[\Delta^{2}\right]}$ and still super-regular. To see that such a subgraph exists, we use a probabilistic argument. For all distinct $i_{1}, i_{2} \in[r]$, let $\tau_{i_{1} i_{2}}: C_{i_{1} i_{2}} \rightarrow\left[\Delta^{2}\right] \times\left[\Delta^{2}\right]$, where each $\tau_{i_{1} i_{2}}(\alpha)$ is chosen independently at random according to the probability distribution $\left(p_{\left(i_{1}, j_{1}\right),\left(i_{2}, j_{2}\right)}\right)_{j_{1}, j_{2} \in\left[\Delta^{2}\right]}$ with

$$
p_{\left(i_{1}, j_{1}\right),\left(i_{2}, j_{2}\right)}:=\frac{e_{H^{\prime}}\left(X_{i_{1}, j_{1}}, X_{i_{2}, j_{2}}\right)}{e_{H^{\prime}}\left(X_{i_{1}}, X_{i_{2}}\right)} \geq \frac{\gamma^{4} n^{\prime}}{2 \Delta^{4} n^{\prime}} \geq \gamma^{5} .
$$

Define $G_{1}$ by keeping each edge $e \in E\left(G\left[V_{i_{1}, j_{1}}, V_{i_{2}, j_{2}}\right]\right)$ if $\tau_{i_{1} i_{2}}(c(e))=\left(j_{1}, j_{2}\right)$. By Lemma 5.1, and since $G\left[V_{i_{1}, j_{1}}, V_{i_{2}, j_{2}}\right]$ is $\left(2 \Delta^{2} \varepsilon, d\right)$-super-regular, there exists $G_{1} \subseteq G$ such that the colouring of $G_{1}$ is colour-split and $G_{1}\left[V_{i_{1}, j_{1}}, V_{i_{2}, j_{2}}\right]$ is $\left(\varepsilon^{\prime} / 2, p_{\left(i_{1}, j_{1}\right),\left(i_{2}, j_{2}\right)} d\right)$-super-regular for all distinct $i_{1}, i_{2} \in[r]$ and all $j_{1}, j_{2} \in\left[\Delta^{2}\right]$. 
For all distinct $i_{1}, i_{2} \in[r]$, all $j_{1}, j_{2} \in\left[\Delta^{2}\right]$, and every colour $\alpha \in C_{i_{1} i_{2}}$, we obtain

$$
\begin{aligned}
e_{G_{1}}^{\alpha}\left(V_{i_{1}, j_{1}}, V_{i_{2}, j_{2}}\right) & \leq e_{G}^{\alpha}\left(V_{i_{1}, j_{1}}, V_{i_{2}, j_{2}}\right) \stackrel{(5.18)}{\leq}\left(1-\frac{3 \gamma}{4}\right) \frac{(d+3 \varepsilon) n^{\prime 2}}{e_{H^{\prime}}\left(X_{i_{1}}, X_{i_{2}}\right)} \\
& =\left(1-\frac{3 \gamma}{4}\right) \frac{p_{\left(i_{1}, j_{1}\right),\left(i_{2}, j_{2}\right)}(d+3 \varepsilon) n^{\prime 2}}{e_{H^{\prime}}\left(X_{i_{1}, j_{1}}, X_{i_{2}, j_{2}}\right)}
\end{aligned}
$$

and thus, since $G_{1}\left[V_{i_{1}, j_{1}}, V_{i_{2}, j_{2}}\right]$ is $\left(\varepsilon^{\prime} / 2, p_{\left(i_{1}, j_{1}\right),\left(i_{2}, j_{2}\right)} d\right)$-super-regular, we conclude that

$$
e_{G_{1}}^{\alpha}\left(V_{i_{1}, j_{1}}, V_{i_{2}, j_{2}}\right) \leq\left(1-\frac{2 \gamma}{3}\right) \frac{e_{G_{1}}\left(V_{i_{1}, j_{1}}, V_{i_{2}, j_{2}}\right)}{e_{H^{\prime}}\left(X_{i_{1}, j_{1}}, X_{i_{2}, j_{2}}\right)} .
$$

Let $G_{2}$ be the spanning subgraph of $G_{1}$ where for each bipartite pair $G_{1}\left[V_{i_{1}, j_{1}}, V_{i_{2}, j_{2}}\right]$, we keep each edge independently at random with probability $d^{\prime} /\left(p_{\left(i_{1}, j_{1}\right),\left(i_{2}, j_{2}\right)} d\right)$. As $G_{1}\left[V_{i_{1}, j_{1}}, V_{i_{2}, j_{2}}\right]$ is $\left(\varepsilon^{\prime} / 2, p_{\left(i_{1}, j_{1}\right),\left(i_{2}, j_{2}\right)} d\right)$-super-regular, we may conclude by simple applications of Chernoff's inequality that with probability at least $1-1 / n$ for all distinct $i_{1}, i_{2} \in[r]$ and all $j_{1}, j_{2} \in\left[\Delta^{2}\right]$, the graph $G_{2}\left[V_{i_{1}, j_{1}}, V_{i_{2}, j_{2}}\right]$ is $\left(\varepsilon^{\prime}, d^{\prime}\right)$-super-regular, and by (5.20) for every colour $\alpha \in C$, we have

$$
e_{G_{2}}^{\alpha}\left(V_{i_{1}, j_{1}}, V_{i_{2}, j_{2}}\right) \leq\left(1-\frac{\gamma}{2}\right) \frac{e_{G_{2}}\left(V_{i_{1}, j_{1}}, V_{i_{2}, j_{2}}\right)}{e_{H^{\prime}}\left(X_{i_{1}, j_{1}}, X_{i_{2}, j_{2}}\right)} .
$$

Finally, we may add edges in the empty bipartite graphs $G_{2}\left[V_{i, j}, V_{i, j^{\prime}}\right]$ for all $i \in[r]$ and all distinct $j, j^{\prime} \in\left[\Delta^{2}\right]$ in such a way that we obtain a supergraph $G^{\prime} \supseteq G_{2}$ where $G^{\prime}\left[V_{i_{1}, j_{1}}, V_{i_{2}, j_{2}}\right]$ is $\left(\varepsilon^{\prime}, d^{\prime}\right)$-super-regular for all $i_{1}, i_{2} \in[r]$ and $j_{1}, j_{2} \in\left[\Delta^{2}\right],\left(i_{1}, j_{1}\right) \neq\left(i_{2}, j_{2}\right)$. Hence, we conclude that $\left(H^{\prime}, G^{\prime},\left(X_{i, j}\right)_{i \in[r], j \in\left[\Delta^{2}\right]},\left(V_{i, j}\right)_{i \in[r], j \in\left[\Delta^{2}\right]}\right)$ is an $\left(\varepsilon^{\prime}, d^{\prime}\right)$-super-regular blow-up instance that satisfies (c).

Let $c^{\text {art }}:\left(\begin{array}{c}V(G) \\ 2\end{array}\right) \rightarrow C^{\text {art }}$ be a rainbow edge-colouring of all possible edges $\left(\begin{array}{c}V(G) \\ 2\end{array}\right)$ such that $C^{\text {art }} \cap C=\emptyset$. By colouring the edges $E\left(G^{\prime}\right) \backslash E\left(G_{2}\right)$ using $c^{\text {art }}$, we may obtain an edge-colouring $c^{\prime}: E\left(G^{\prime}\right) \rightarrow C \cup C^{\text {art }}$ that extends $c$ and is clearly $\Lambda$-bounded. By the construction of $G_{2}$, the colouring $c^{\prime}$ is colour-split, and

$$
\left(1-\frac{\gamma}{2}\right) \frac{e_{G^{\prime}}\left(V_{i_{1}, j_{1}}, V_{i_{2}, j_{2}}\right)}{e_{H^{\prime}}\left(X_{i_{1}, j_{1}}, X_{i_{2}, j_{2}}\right)} \text {-bounded }
$$

for each bipartite subgraph $G^{\prime}\left[V_{i_{1}, j_{1}}, V_{i_{2}, j_{2}}\right]$ with $i_{1}, i_{2} \in[r], j_{1}, j_{2} \in\left[\Delta^{2}\right],\left(i_{1}, j_{1}\right) \neq\left(i_{2}, j_{2}\right)$ due to (5.21). This yields (d) and completes the proof.

\section{Approximate Embedding Lemma}

In this section, we prove the 'Approximate Embedding Lemma' (Lemma 6.3), which allows us to embed a cluster $X_{i}$ into $V_{i}$ (here $X_{0}, V_{0}$ ) almost completely while maintaining crucial properties of other clusters for future embedding rounds. As outlined in Section 3, we track these properties using 'candidacy graphs' $A_{i}$, which are auxiliary bipartite graphs between $X_{i}$ and $V_{i}$ where $x v \in E\left(A_{i}\right)$ only if $v$ is still a suitable image for $x$ given previous embedding rounds.

We say that $\left(H, G,\left(A_{i}\right)_{i \in[r]_{0}}, c\right)$ is an embedding-instance if

○ $H, G$ are graphs and $A_{i}$ is a bipartite graph with vertex partition $\left(X_{i}, V_{i}\right)$ for every $i \in[r]_{0}$ such that $\left(X_{i}\right)_{i \in[r]_{0}}$ is a partition of $V(H)$ into independent sets, $\left(V_{i}\right)_{i \in[r]_{0}}$ is a partition of $V(G)$, and $\left|X_{i}\right|=\left|V_{i}\right|$ for all $i \in[r]_{0}$.

- For all $i \in[r]$, the graph $H\left[X_{0}, X_{i}\right]$ is a matching.

$\circ c: E\left(G \cup \bigcup_{i \in[r]_{0}} A_{i}\right) \rightarrow 2^{C}$ is an edge set colouring that is colour-split with respect to the partition $\left(X_{0}, \ldots, X_{r}, V_{0}, \ldots, V_{r}\right)$ and satisfies $|c(e)|=1$ for all $e \in E(G)$. 


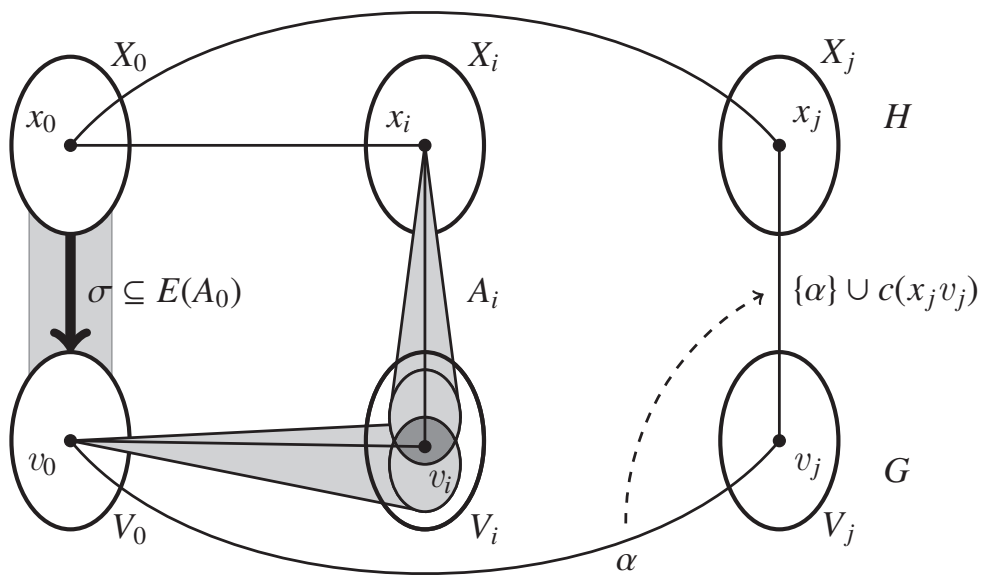

Figure 1. If $x_{0}$ is mapped to $v_{0}$ by $\sigma$, then only those candidates of $x_{i}$ remain that are neighbours of $v_{0}$. Moreover, colour $\alpha$ of the edge $v_{0} v_{j}$ is added to the candidate edge $x_{j} v_{j}$, which captures the information that if $x_{j}$ is later embedded at $v_{j}$, then this embedding uses $\alpha$.

We say that $\left(H, G,\left(A_{i}\right)_{i \in[r]_{0}}, c\right)$ is an $\left(\varepsilon,\left(d_{i}^{G}\right)_{i \in[r]},\left(d_{i}\right)_{i \in[r]_{0}}, t, \Lambda\right)$-embedding-instance if, in addition, we have that

○ $G\left[V_{0}, V_{i}\right]$ is $\left(\varepsilon, d_{i}^{G}\right)$-super-regular and $c$ restricted to $G\left[V_{0}, V_{i}\right]$ is $(1+\varepsilon) e_{G}\left(V_{0}, V_{i}\right) / e_{H}\left(X_{0}, X_{i}\right)$ bounded for all $i \in[r]$.

○ $A_{i}$ is $\left(\varepsilon, d_{i}\right)$-super-regular and $c$ restricted to $A_{i}$ is $(1+\varepsilon) d_{i}\left|X_{i}\right|$-bounded for all $i \in[r]_{0}$.

$\circ c$ is locally $\Lambda$-bounded and $|c(e)| \leq t$ for all $e \in \bigcup_{i \in[r]_{0}} E\left(A_{i}\right)$.

Here, $X_{0}$ is the cluster we want to embed into $V_{0}$ by finding an almost perfect rainbow matching $\sigma$ in $A_{0}$, and $t$ can be thought of as the number of clusters we have previously embedded. For a matching $\sigma$, we denote by $V(\sigma)$ the vertices contained in $\sigma$, and for convenience, we identify matchings $\sigma$ between $X_{0}$ and $V_{0}$ with functions $\sigma: X_{0}^{\sigma} \rightarrow V_{0}^{\sigma}$, where $X_{0}^{\sigma}=V(\sigma) \cap X_{0}$ and $V_{0}^{\sigma}=V(\sigma) \cap V_{0}$. Whenever we write $x v \in E\left(A_{i}\right)$, we tacitly assume that $x \in X_{i}$ and $v \in V_{i}$.

The following two definitions encapsulate how the choice of $\sigma$ affects the candidacy graphs $\left(A_{i}\right)_{i \in[r]}$ and their colouring for the next step (see Figure 1). Let $\left(H, G,\left(A_{i}\right)_{i \in[r]_{0}}, c\right)$ be an embedding-instance.

Definition 6.1 (Updated candidacy graphs). For a matching $\sigma: X_{0}^{\sigma} \rightarrow V_{0}^{\sigma}$ in $A_{0}$, we define $\left(A_{i}^{\sigma}\right)_{i \in[r]}$ as the updated candidacy graphs (with respect to $\sigma$ ) as follows: for every $i \in[r]$, let $A_{i}^{\sigma}$ be the spanning subgraph of $A_{i}$ containing precisely those edges $x v \in E\left(A_{i}\right)$ for which the following holds: if $x$ has an $H$-neighbour $x_{0} \in X_{0}^{\sigma}$ (which would be unique), then $\sigma\left(x_{0}\right) v \in E\left(G\left[V_{0}, V_{i}\right]\right)$.

This definition ensures that when we embed $x$ in a future round, we are guaranteed that the $H$-edge $x_{0} x$ is mapped to a $G$-edge. Note that this definition does not depend at all on the colouring $c$. Moreover, we also define updated colourings for the updated candidacy graphs, where we add up to one additional colour to the edges in the new candidacy graphs according to $\sigma$.

Definition 6.2 (Updated colouring). For a matching $\sigma: X_{0}^{\sigma} \rightarrow V_{0}^{\sigma}$ in $A_{0}$, we define the updated edge set colouring $c^{\sigma}$ of the updated candidacy graphs as follows: for each $i \in[r]$ and $x v \in E\left(A_{i}^{\sigma}\right)$, when $x$ has an $H$-neighbour $x_{0} \in X_{0}^{\sigma}$, then set $c^{\sigma}(x v):=c(x v) \cup c\left(\sigma\left(x_{0}\right) v\right)$, and otherwise set $c^{\sigma}(x v):=c(x v)$.

We now state and prove our Approximate Embedding Lemma.

Lemma 6.3 (Approximate Embedding Lemma). Let

$$
1 / n \ll \varepsilon \ll \varepsilon^{\prime} \ll\left(d_{i}^{G}\right)_{i \in[r]},\left(d_{i}\right)_{i \in[r]_{0}}, 1 / \Lambda, 1 / r, 1 /(t+1) .
$$


Suppose $\left(H, G,\left(A_{i}\right)_{i \in[r]_{0}}, c\right)$ is an $\left(\varepsilon,\left(d_{i}^{G}\right)_{i \in[r]},\left(d_{i}\right)_{i \in[r]_{0}}, t, \Lambda\right)$-embedding-instance with $\left|V_{0}\right|=n$, $\left|V_{i}\right|=(1 \pm \varepsilon) n$, and $e_{H}\left(X_{0}, X_{i}\right) \geq \varepsilon^{\prime} n$ for all $i \in[r]$. Suppose the codegree of $c$ is $K \leq \sqrt{n}$.

Then there is a rainbow matching $\sigma: X_{0}^{\sigma} \rightarrow V_{0}^{\sigma}$ in $A_{0}$ of size at least $\left(1-\varepsilon^{\prime}\right) n$ such that for all $i \in[r]$, there exists a spanning subgraph $A_{i}^{\text {new }}$ of the updated candidacy graph $A_{i}^{\sigma}$ and

(I) $6.3 A_{i}^{\text {new }}$ is $\left(\varepsilon^{\prime}, d_{i}^{G} d_{i}\right)$-super-regular.

(II) $)_{6.3}$ The updated colouring $c^{\sigma}$ restricted to $A_{i}^{\text {new }}$ is $\left(1+\varepsilon^{\prime}\right) d_{i}^{G} d_{i}\left|X_{i}\right|$-bounded.

(III) $6.3 c^{\sigma}$ restricted to $A_{i}^{\text {new }}$ has codegree at most $\max \left\{K, n^{\varepsilon}\right\}$.

We split the proof into three steps. In Step 1, we remove non-typical vertices and edges to guarantee that certain neighbourhoods intersect appropriately. In Step 2, we use a suitable hypergraph construction together with Theorem 4.3 to obtain the required rainbow matching $\sigma$. By defining certain weight functions in Step 3, we utilise the conclusions of Theorem 4.3 to show that $\sigma$ can be chosen such that (I) $6.3-(\mathrm{III})_{6.3}$ hold.

Proof. Without loss of generality, we may assume that $|c(e)|=t$ for all $e \in E\left(A_{0}\right)$. (Otherwise, we may simply add new 'dummy' colours in such a way that the obtained colouring still satisfies the conditions of the lemma, and these colours can simply be deleted afterward.)

We also choose a new constant $\hat{\varepsilon}$ such that $\varepsilon \ll \hat{\varepsilon} \ll \varepsilon^{\prime}$.

Step 1. Removing non-typical vertices and edges

In this step, we define subgraphs of $G$ and $\left(A_{i}\right)_{i \in[r]_{0}}$ to achieve that certain neighbourhoods intersect appropriately (see properties (6.4)-(6.6)). Let $H^{+}$be an auxiliary supergraph of $H$ that is obtained by adding a maximal number of edges between $X_{0}$ and $X_{i}$ for every $i \in[r]$ subject to $H^{+}\left[X_{0}, X_{i}\right]$ being a matching (note that $e_{H^{+}}\left(X_{0}, X_{i}\right) \geq(1-\varepsilon) n$ ).

Let $A_{0}^{\text {bad }}$ be the spanning subgraph of $A_{0}$ such that an edge $x_{0} v_{0} \in E\left(A_{0}\right)$ belongs to $A_{0}^{\text {bad }}$ if there is some $i \in[r]$ with $\left\{x_{i}\right\}=N_{H^{+}}\left(x_{0}\right) \cap X_{i}$ and

$$
\left|N_{A_{i}}\left(x_{i}\right) \cap N_{G}\left(v_{0}\right)\right| \neq\left(d_{i}^{G} d_{i} \pm 3 \varepsilon\right)\left|V_{i}\right|
$$

For $i \in[r]$, let $A_{i}^{b a d}$ be the spanning subgraph of $A_{i}$ such that an edge $x_{i} v_{i} \in E\left(A_{i}\right)$ belongs to $A_{i}^{b a d}$ if $\left\{x_{0}\right\}=N_{H^{+}}\left(x_{i}\right) \cap X_{0}$ and

$$
\left|N_{A_{0}}\left(x_{0}\right) \cap N_{G}\left(v_{i}\right)\right| \neq\left(d_{i}^{G} d_{0} \pm 3 \varepsilon\right)\left|V_{0}\right|
$$

Let $G^{\text {bad }}$ be the spanning subgraph of $G$ such that an edge $v_{0} v_{i} \in E\left(G\left[V_{0}, V_{i}\right]\right)$ belongs to $G^{b a d}\left[V_{0}, V_{i}\right]$ for $i \in[r]$ whenever

$$
e_{H}\left(N_{A_{0}}\left(v_{0}\right), N_{A_{i}}\left(v_{i}\right)\right) \neq\left(d_{0} d_{i} \pm 3 \varepsilon\right) e_{H}\left(X_{0}, X_{i}\right) \text {. }
$$

Using Fact 4.4, it is easy to see that $\Delta\left(A_{0}^{\text {bad }}\right) \leq 3 r \varepsilon n$ and $\Delta\left(A_{i}^{\text {bad }}\right) \leq 3 \varepsilon\left|V_{i}\right|$ for each $i \in[r]$. We also claim that for each $i \in[r]_{0}$, there exists $V_{i}^{\text {bad }} \subseteq V_{i}$ with $\left|V_{i}^{b a d}\right| \leq 3 r \varepsilon n$ such that all vertices not in $V_{0}^{b a d} \cup \cdots \cup V_{r}^{b a d}$ have degree at most $3 r \varepsilon n$ in $G^{b a d}$. Indeed, fix $i \in[r]$ and let $\widetilde{X}_{0}:=N_{H}\left(X_{i}\right)$ and $\widetilde{X}_{i}:=N_{H}\left(X_{0}\right)$. Recall that $\left|\widetilde{X}_{0}\right|=\left|\widetilde{X}_{i}\right|=e_{H}\left(X_{0}, X_{i}\right) \geq \varepsilon^{\prime} n$. Using Fact 4.4, there exists $V_{i}^{\text {bad }} \subseteq V_{i}$ with $\left|V_{i}^{\text {bad }}\right| \leq 3 \varepsilon\left|V_{i}\right|$ such that all $v_{i} \in V_{i} \backslash V_{i}^{\text {bad }}$ satisfy $\left|N_{A_{i}}\left(v_{i}\right) \cap \widetilde{X}_{i}\right|=\left(d_{i} \pm \varepsilon\right)\left|\widetilde{X}_{i}\right|$. Now, fix such a vertex $v_{i}$. Let $U:=N_{H}\left(N_{A_{i}}\left(v_{i}\right)\right)$. Using Fact 4.4 again, we can see that all but at most $3 \varepsilon n$ vertices $v_{0} \in V_{0}$ satisfy $\left|N_{A_{0}}\left(v_{0}\right) \cap U\right|=\left(d_{0} \pm \varepsilon\right)|U|=\left(d_{0} \pm \varepsilon\right)\left(d_{i} \pm \varepsilon\right) e_{H}\left(X_{0}, X_{i}\right)$. Hence, $\operatorname{deg}_{G^{\text {bad }}}\left(v_{i}\right) \leq 3 \varepsilon n$. Similarly, one can see that there exists $V_{0, i}^{\text {bad }} \subseteq V_{0}$ with $\left|V_{0, i}^{\text {bad }}\right| \leq 3 \varepsilon n$ such that all $v_{0} \in V_{0} \backslash V_{0, i}^{b a d}$ satisfy $\left|N_{G^{b a d}}\left(v_{0}\right) \cap V_{i}\right| \leq 3 \varepsilon n$. Let $V_{0}^{\text {bad }}:=\bigcup_{i=1}^{r} V_{0, i}^{b a d}$. Then $V_{0}^{\text {bad }}, \ldots, V_{r}^{\text {bad }}$ are as desired. 
Now, let

$$
\begin{aligned}
A_{0}^{\prime} & :=A_{0}\left[X_{0}, V_{0} \backslash V_{0}^{b a d}\right]-E\left(A_{0}^{b a d}\right), \quad A_{i}^{\prime}:=A_{i}-E\left(A_{i}^{b a d}\right), \\
G_{0 i}^{\prime} & :=G\left[V_{0} \backslash V_{0}^{b a d}, V_{i}\right]-E\left(G^{b a d}\left[V_{0}, V_{i} \backslash V_{i}^{b a d}\right]\right), \quad \text { for all } i \in[r] .
\end{aligned}
$$

Since we only seek an almost perfect rainbow matching $\sigma$ in $A_{0}$, we can remove the vertices $V_{0}^{b a d}$ from $A_{0}$ and find $\sigma$ in $A_{0}^{\prime}$. By keeping the vertices $V_{i}^{b a d}$ for $i \in[r]$ and the corresponding edges $E\left(G\left[V_{0}, V_{i}^{b a d}\right]\right)$ in $G_{0 i}^{\prime}$, we can guarantee that the candidacy graphs $A_{i}^{\prime}$ are still spanning subgraphs of $A_{i}$.

By Fact 4.5 , we have that $G_{0 i}^{\prime}$ is $\left(\hat{\varepsilon}, d_{i}^{G}\right)$-super-regular, that $A_{0}^{\prime}$ is $\left(\hat{\varepsilon}, d_{0}\right)$-super-regular, and that $A_{i}^{\prime}$ is $\left(\hat{\varepsilon}, d_{i}\right)$-super-regular. Crucially, we now have the following properties:

$$
\begin{aligned}
& \left|N_{A_{i}^{\prime}}\left(x_{i}\right) \cap N_{G_{0 i}^{\prime}}\left(v_{0}\right)\right|=\left(d_{i}^{G} d_{i} \pm \hat{\varepsilon}\right)\left|V_{i}\right|, \\
& \text { for all } x_{0} v_{0} \in E\left(A_{0}^{\prime}\right) \text { whenever }\left\{x_{i}\right\}=N_{H^{+}}\left(x_{0}\right) \cap X_{i}, i \in[r] ; \\
& \left|N_{A_{0}^{\prime}}\left(x_{0}\right) \cap N_{G_{0 i}^{\prime}}\left(v_{i}\right)\right|=\left(d_{i}^{G} d_{0} \pm \hat{\varepsilon}\right)\left|V_{0}\right|, \\
& \text { for all } x_{i} v_{i} \in E\left(A_{i}^{\prime}\right), i \in[r], \text { whenever }\left\{x_{0}\right\}=N_{H^{+}}\left(x_{i}\right) \cap X_{0} ; \\
& e_{H}\left(N_{A_{0}^{\prime}}\left(v_{0}\right), N_{A_{i}^{\prime}}\left(v_{i}\right)\right)=\left(d_{0} d_{i} \pm \hat{\varepsilon}\right) e_{H}\left(X_{0}, X_{i}\right), \\
& \text { for all } v_{0} v_{i} \in E\left(G_{0 i}^{\prime}-V_{i}^{b a d}\right) \text { and } i \in[r] .
\end{aligned}
$$

Indeed, consider $x_{0} v_{0} \in E\left(A_{0}^{\prime}\right)$ with $\left\{x_{i}\right\}=N_{H^{+}}\left(x_{0}\right) \cap X_{i}$. By (6.1), we have $\left|N_{A_{i}}\left(x_{i}\right) \cap N_{G}\left(v_{0}\right)\right|=$ $\left(d_{i}^{G} d_{i} \pm 3 \varepsilon\right)\left|V_{i}\right|$. Moreover, $v_{0} \notin V_{0}^{\text {bad }}$. Hence, $\operatorname{deg}_{A_{i}^{\text {bad }}}\left(x_{i}\right), \operatorname{deg}_{G^{\text {bad }}}\left(v_{0}\right) \leq 3 r \varepsilon n$, which implies (6.4). Similar arguments hold for (6.5) and (6.6).

Step 2. Constructing an auxiliary hypergraph

We aim to apply Theorem 4.3 to find the required rainbow matching $\sigma$. To this end, let $f_{e}:=e \cup c(e)$ for $e \in E\left(A_{0}^{\prime}\right)$ and let $\mathcal{H}$ be the $(t+2)$-uniform hypergraph $\mathcal{H}$ with vertex set $X_{0} \cup V_{0} \cup C$ and edge set $\left\{f_{e}: e \in E\left(A_{0}^{\prime}\right)\right\}$. A key property of the construction of $\mathcal{H}$ is a bijection between rainbow matchings $M$ in $A_{0}^{\prime}$ and matchings $\mathcal{M}$ in $\mathcal{H}$ by assigning $M$ to $\mathcal{M}=\left\{f_{e}: e \in M\right\}$.

To apply Theorem 4.3, we first establish upper bounds on $\Delta(\mathcal{H})$ and $\Delta^{c}(\mathcal{H})$. Since $A_{0}^{\prime}$ is $\left(\hat{\varepsilon}, d_{0}\right)$ super-regular, $\left|X_{0}\right|=n$, and $c$ restricted to $A_{0}^{\prime}$ is $(1+\varepsilon) d_{0} n$-bounded, we conclude that

$$
\Delta(\mathcal{H}) \leq\left(d_{0}+\hat{\varepsilon}\right) n
$$

Let $\Delta:=\left(d_{0}+\hat{\varepsilon}\right) n$. Since $c$ is locally $\Lambda$-bounded, the codegree in $\mathcal{H}$ of a vertex in $X_{0} \cup V_{0}$ and a colour in $C$ is at most $\Lambda$. By assumption, the codegree in $\mathcal{H}$ of two colours in $C$ is at most $K$. For two vertices in $X_{0} \cup V_{0}$, the codegree in $\mathcal{H}$ is at most 1 . Altogether, this implies that

$$
\Delta^{c}(\mathcal{H}) \leq \sqrt{n} \leq \Delta^{1-\varepsilon^{2}}
$$

Suppose $\mathcal{W}$ is a set of given weight functions $\omega: E\left(A_{0}^{\prime}\right) \rightarrow[\Lambda]_{0}$ with $|\mathcal{W}| \leq n^{5}$ (which we will explicitly specify in Step 3 to establish $\left.(\mathrm{I})_{6.3}-(\mathrm{III})_{6.3}\right)$. Note that every weight function $\omega: E\left(A_{0}^{\prime}\right) \rightarrow[\Lambda]_{0}$ naturally corresponds to a weight function $\omega_{\mathcal{H}}: E(\mathcal{H}) \rightarrow[\Lambda]_{0}$ by defining $\omega_{\mathcal{H}}\left(f_{e}\right):=\omega(e)$. If $\omega\left(E\left(A_{0}^{\prime}\right)\right) \geq n^{1+\varepsilon / 2}$, define $\tilde{\omega}:=\omega$. Otherwise, arbitrarily choose $\tilde{\omega}: E\left(A_{0}^{\prime}\right) \rightarrow[\Lambda]_{0}$ such that $\omega \leq \tilde{\omega}$ and $\tilde{\omega}\left(E\left(A_{0}^{\prime}\right)\right)=n^{1+\varepsilon / 2}$. By (6.7) and (6.8), we can apply Theorem 4.3 (with $\left(d_{0}+\hat{\varepsilon}\right) n, \varepsilon^{2}$, $t+2,\left\{\tilde{\omega}_{\mathcal{H}}\right\} \omega \in \mathcal{W}$ playing the roles of $\left.\Delta, \delta, r, \mathcal{W}\right)$ to obtain a matching $\mathcal{M}$ in $\mathcal{H}$ that corresponds to a 
rainbow matching $M$ in $A_{0}^{\prime}$ that satisfies the following property by the conclusion of Theorem 4.3:

$$
\begin{aligned}
& \omega(M)=\left(1 \pm \hat{\varepsilon}^{1 / 2}\right) \frac{\omega\left(E\left(A_{0}^{\prime}\right)\right)}{d_{0} n}, \text { for all } \omega \in \mathcal{W} \text { with } \omega\left(E\left(A_{0}^{\prime}\right)\right) \geq n^{1+\varepsilon / 2} \\
& \omega(M) \leq \max \left\{\left(1+\hat{\varepsilon}^{1 / 2}\right) \frac{\omega\left(E\left(A_{0}^{\prime}\right)\right)}{d_{0} n}, n^{\varepsilon}\right\} \text { for all } \omega \in \mathcal{W} .
\end{aligned}
$$

Let $\sigma: X_{0}^{\sigma} \rightarrow V_{0}^{\sigma}$ be the function given by the matching $M$, where $X_{0}^{\sigma}=X_{0} \cap V(M)$ and $V_{0}^{\sigma}=$ $V_{0} \cap V(M)$.

One way to exploit (6.9) is to control the number of edges in $M$ between sufficiently large sets of vertices. To this end, for subsets $S \subseteq X_{0}$ and $T \subseteq V_{0}$ such that $|S|,|T| \geq 2 \hat{\varepsilon} n$, we define a weight function $\omega_{S, T}: E\left(A_{0}^{\prime}\right) \rightarrow[\Lambda]_{0}$ with

$$
\omega_{S, T}(e):= \begin{cases}1 & \text { if } e \in E\left(A_{0}^{\prime}\left[S, T \backslash V_{0}^{b a d}\right]\right) \\ 0 & \text { otherwise. }\end{cases}
$$

That is, $\omega_{S, T}(M)$ counts the number of edges between $S$ and $T$ that lie in $M$. Since $A_{0}^{\prime}$ is $\left(\hat{\varepsilon}, d_{0}\right)$-superregular, (6.9) implies (whenever $\omega_{S, T} \in \mathcal{W}$ ) that

$$
\left|\sigma\left(S \cap X_{0}^{\sigma}\right) \cap T\right|=\omega_{S, T}(M) \stackrel{(6.9)}{=}\left(1 \pm \hat{\varepsilon}^{1 / 2}\right) \frac{e\left(A_{0}^{\prime}\left[S, T \backslash V_{0}^{\text {bad }}\right]\right)}{d_{0} n}=\left(1 \pm 2 \hat{\varepsilon}^{1 / 2}\right) \frac{|S||T|}{n}
$$

Step 3. Employing weight functions to conclude (I) $6.3-(\mathrm{III})_{6.3}$

By Step 2, we may assume that (6.9) holds for a set of weight functions $\mathcal{W}$ that we will define during this step. We will show that for this choice of $\mathcal{W}$, the matching $\sigma: X_{0}^{\sigma} \rightarrow V_{0}^{\sigma}$ as obtained in Step 1 satisfies $(\mathrm{I})_{6.3}-(\mathrm{III})_{6.3}$. Similar as in Definition 6.1 (here with $H$ replaced by $H^{+}$), we define subgraphs $\left(A_{i}^{*}\right)_{i \in[r]}$ of $\left(A_{i}^{\prime}\right)_{i \in[r]}$ as follows. For every $i \in[r]$, let $A_{i}^{*}$ be the spanning subgraph of $A_{i}^{\prime}$ containing precisely those edges $x v \in E\left(A_{i}^{\prime}\right)$ for which the following holds: if $\left\{x_{0}\right\}=N_{H^{+}}(x) \cap X_{0}^{\sigma}$, then $\sigma\left(x_{0}\right) v \in E\left(G_{0 i}^{\prime}\right)$. Since $A_{i}^{\prime} \subseteq A_{i}$, and due to the construction of $A_{i}^{*}$, we conclude that $A_{i}^{*}$ is a spanning subgraph of the updated candidacy graph $A_{i}^{\sigma}$ (with respect to $\sigma$ ) for every $i \in[r]$ (see Definition 6.1). By taking a suitable subgraph of $A_{i}^{*}$, we will later obtain the required candidacy graph $A_{i}^{\text {new }}$.

First, we show that the matching $M$ has size at least $\left(1-2 \hat{\varepsilon}^{1 / 2}\right) n$. Adding $\omega_{X_{0}, V_{0}}$ as defined in (6.11) to $\mathcal{W}$ and using (6.12) yields

$$
|M| \geq\left(1-2 \hat{\varepsilon}^{1 / 2}\right) n
$$

For every $i \in[r]$, define $X_{i}^{H}:=N_{H^{+}}\left(X_{0}^{\sigma}\right) \cap X_{i}$. Note that $\left|X_{i}^{H}\right|=\left(1 \pm 3 \hat{\varepsilon}^{1 / 2}\right)\left|X_{i}\right|=\left(1 \pm 4 \hat{\varepsilon}^{1 / 2}\right) n$.

Step 3.1. Checking $(\mathrm{I})_{6.3}$

To prove $(\mathrm{I})_{6.3}$, we first show that $A_{i}^{*}\left[X_{i}^{H}, V_{i}\right]$ is super-regular for every $i \in[r]$. We will show that every vertex in $X_{i}^{H} \cup V_{i}$ has the appropriate degree and that the common neighbourhood of most pairs of vertices in $V_{i}$ has the correct size, such that we can employ Theorem 4.6 to guarantee the super-regularity of $A_{i}^{*}\left[X_{i}^{H}, V_{i}\right]$.

For all $i \in[r]$ and for every vertex $x \in X_{i}^{H}$ with $\left\{x_{0}\right\}=N_{H^{+}}(x) \cap X_{0}^{\sigma}$, we have $\operatorname{deg}_{A_{i}^{*}\left[X_{i}^{H}, V_{i}\right]}(x)=$ $\left|N_{A_{i}^{\prime}}(x) \cap N_{G_{0 i}^{\prime}}\left(\sigma\left(x_{0}\right)\right)\right|$. Hence, (6.4) implies that

$$
\operatorname{deg}_{A_{i}^{*}\left[X_{i}^{H}, V_{i}\right]}(x)=\left(d_{i}^{G} d_{i} \pm \hat{\varepsilon}\right)\left|V_{i}\right| .
$$


For $v \in V_{i}$, let $U_{v}:=N_{A_{i}^{\prime}}(v) \subseteq X_{i}$. Observe that

$$
\operatorname{deg}_{A_{i}^{*}\left[X_{i}^{H}, V_{i}\right]}(v)=\left|\sigma\left(N_{H^{+}}\left(U_{v}\right) \cap X_{0}^{\sigma}\right) \cap N_{G_{0 i}^{\prime}}(v)\right|,
$$

and $\left|N_{H^{+}}\left(U_{v}\right) \cap X_{0}\right|=\left|N_{A_{i}^{\prime}}(v)\right| \pm \varepsilon n=\left(d_{i} \pm 2 \hat{\varepsilon}\right) n$, and $\left|N_{G_{0 i}^{\prime}}(v)\right|=\left(d_{i}^{G} \pm 2 \hat{\varepsilon}\right) n$. Adding for every $i \in[r]$ and every vertex $v \in V_{i}$, the weight function $\omega_{S, T}$ as defined in (6.11) for $S:=N_{H^{+}}\left(U_{v}\right) \cap X_{0}$ and $T:=N_{G_{0 i}^{\prime}}(v)$ to $\mathcal{W}$, we obtain that

$$
\begin{aligned}
\operatorname{deg}_{A_{i}^{*}\left[X_{i}^{H}, V_{i}\right]}(v) \stackrel{(6.12),(6.14)}{=}\left(1 \pm 2 \hat{\varepsilon}^{1 / 2}\right)\left|N_{H^{+}}\left(U_{v}\right) \cap X_{0}\right|\left|N_{G_{0 i}^{\prime}}(v)\right| n^{-1} \\
=\quad\left(d_{i}^{G} d_{i} \pm \hat{\varepsilon}^{1 / 3}\right)\left|X_{i}^{H}\right|
\end{aligned}
$$

Note that these are at most $2 r n$ weight functions $\omega_{S, T}$ that we added to $\mathcal{W}$.

We will use Theorem 4.6 to show that $A_{i}^{*}\left[X_{i}^{H}, V_{i}\right]$ is super-regular. We call a pair of vertices $u, v \in V_{i}$ $\operatorname{good}$ if $\left|N_{A_{i}^{\prime}}(u, v)\right|=\left(d_{i} \pm \hat{\varepsilon}\right)^{2}\left|X_{i}\right|$ and $\left|N_{G_{0 i}^{\prime}}(u, v)\right|=\left(d_{i}^{G} \pm \hat{\varepsilon}\right)^{2} n$. By the $\hat{\varepsilon}$-regularity of $A_{i}^{\prime}$ and $G_{0 i}^{\prime}$, using Fact 4.4, there are at most $2 \hat{\varepsilon}\left|V_{i}\right|^{2}$ pairs $u, v \in V_{i}$ that are not good. For every $i \in[r]$ and all good pairs $u, v \in V_{i}$, let $S_{u, v}:=N_{H^{+}}\left(N_{A_{i}^{\prime}}(u, v)\right) \cap X_{0}$ and $T_{u, v}:=N_{G_{0 i}^{\prime}}(u, v)$. We add the weight function $\omega_{S_{u, v}, T_{u, v}}$ as defined in (6.11) to $\mathcal{W}$. Observe that $\left|S_{u, v}\right|=\left|N_{A_{i}^{\prime}}(u, v)\right| \pm \varepsilon n=\left(d_{i} \pm 2 \hat{\varepsilon}\right)^{2}\left|X_{i}\right|$ and $\left|T_{u, v}\right|=\left(d_{i}^{G} \pm \hat{\varepsilon}\right)^{2} n$. Note that these are at most $r n^{2}$ functions $\omega_{S_{u, v}, T_{u, v}}$ that we add to $\mathcal{W}$ in this way. By (6.12), we obtain for all good pairs $u, v \in V_{i}$ that

$$
\begin{aligned}
\left|N_{A_{i}^{*}\left[X_{i}^{H}, V_{i}\right]}(u, v)\right| & =\left|\sigma\left(S_{u, v} \cap X_{0}^{\sigma}\right) \cap T_{u, v}\right|=\left(1 \pm 2 \hat{\varepsilon}^{1 / 2}\right)\left|S_{u, v}\right|\left|T_{u, v}\right| n^{-1} \\
& \leq\left(d_{i}^{G} d_{i}+\hat{\varepsilon}^{1 / 3}\right)^{2}\left|X_{i}^{H}\right|
\end{aligned}
$$

Together with (6.15), we can apply Theorem 4.6 and obtain that

$$
A_{i}^{*}\left[X_{i}^{H}, V_{i}\right] \text { is }\left(\hat{\varepsilon}^{1 / 18}, d_{i}^{G} d_{i}\right) \text {-super-regular for every } i \in[r]
$$

To complete the proof of (I) 6.3 , for every $i \in[r]$, since $\left|X_{i} \backslash X_{i}^{H}\right| \leq 3 \hat{\varepsilon}^{1 / 2}\left|X_{i}\right|$, we can easily find a spanning subgraph $A_{i}^{\text {new }}$ of $A_{i}^{*}$ that is $\left(\varepsilon^{\prime}, d_{i}^{G} d_{i}\right)$-super-regular by deleting from every vertex $x \in X_{i} \backslash X_{i}^{H}$ a suitable number of edges. This establishes (I) 6.3 .

Step 3.2. Checking (II) 6.3

Next, we show that for every $i \in[r]$, the edge set colouring $c^{\sigma}$ restricted to $A_{i}^{*}$ is $\left(1+\varepsilon^{\prime}\right) d_{i}^{G} d_{i}\left|X_{i}\right|$ bounded, which implies (II) 6.3 because $A_{i}^{\text {new }} \subseteq A_{i}^{*}$. Recall that we defined $c^{\sigma}$ (in Definition 6.2) such that for $x v \in E\left(A_{i}^{*}\right)$, we have $c^{\sigma}(x v)=c(x v) \cup c\left(\sigma\left(x_{0}\right) v\right)$ if $x$ has an $H$-neighbour $x_{0} \in X_{0}^{\sigma}$, and otherwise $c^{\sigma}(x v)=c(x v)$. Since $c$ is colour-split, we may assume that $c_{A_{i}^{\prime}}: E\left(A_{i}^{\prime}\right) \rightarrow 2^{C_{A_{i}^{\prime}}}$ is the edge set colouring $c$ restricted to $A_{i}^{\prime}$ and $c_{G_{0 i}^{\prime}}: E\left(G_{0 i}^{\prime}\right) \rightarrow C_{G_{0 i}^{\prime}}$ is the edge-colouring $c$ restricted to $G_{0 i}^{\prime}$ such that $C_{A_{i}^{\prime}} \cap C_{G_{0 i}^{\prime}}=\emptyset$ for all $i \in[r]$. Fix $i \in[r]$. We have to show that for all $\alpha \in C_{A_{i}} \cup C_{G_{0 i}^{\prime}}$, there are at most $\left(1+\varepsilon^{\prime}\right) d_{i}^{G} d_{i}\left|X_{i}\right|$ edges of $A_{i}^{*}$ on which $\alpha$ appears.

First, consider $\alpha \in C_{A_{i}^{\prime}}$. Let $E_{\alpha} \subseteq E\left(A_{i}^{\prime}\right)$ be the edges of $A_{i}^{\prime}$ on which $\alpha$ appears. By assumption, $\left|E_{\alpha}\right| \leq(1+\varepsilon) d_{i}\left|X_{i}\right|$. We need to show that $\left|E_{\alpha} \cap E\left(A_{i}^{*}\right)\right| \leq\left(1+\varepsilon^{\prime}\right) d_{i}^{G} d_{i}\left|X_{i}\right|$. To this end, we define a weight function $\omega_{\alpha}: E\left(A_{0}^{\prime}\right) \rightarrow[\Lambda]_{0}$ by setting

$$
\omega_{\alpha}(x v):=\left|\left\{v_{i} \in N_{G_{0 i}^{\prime}}(v): x_{i} v_{i} \in E_{\alpha}, x x_{i} \in E\left(H^{+}\left[X_{0}, X_{i}\right]\right)\right\}\right|
$$


for every $x v \in E\left(A_{0}^{\prime}\right)$, and we add $\omega_{\alpha}$ to $\mathcal{W}$. Note that

$$
\begin{aligned}
\left|E_{\alpha} \cap E\left(A_{i}^{*}\right)\right| & \leq \sum_{x_{i} \in X_{i}^{H}}\left|\left\{v_{i} \in N_{G_{0 i}^{\prime}}(\sigma(x))\right\} x_{i} v_{i} \in E_{\alpha}, x x_{i} \in E\left(H^{+}\left[X_{0}, X_{i}\right]\right)\right|+\Lambda\left|X_{i} \backslash X_{i}^{H}\right| \\
& \leq \omega_{\alpha}(M)+3 \hat{\varepsilon}^{1 / 2} \Lambda\left|X_{i}\right| .
\end{aligned}
$$

We now obtain an upper bound for $\omega_{\alpha}(M)$ using (6.10). For every edge $x_{i} v_{i} \in E_{\alpha}$ with $x x_{i} \in$ $E\left(H^{+}\left[X_{0}, X_{i}\right]\right)$, condition (6.5) states that

$$
\left|N_{A_{0}^{\prime}}(x) \cap N_{G_{0 i}^{\prime}}\left(v_{i}\right)\right|=\left(d_{i}^{G} d_{0} \pm \hat{\varepsilon}\right) n .
$$

Hence, every such edge contributes weight $\left(d_{i}^{G} d_{0} \pm \hat{\varepsilon}\right) n$ to $\omega_{\alpha}\left(E\left(A_{0}^{\prime}\right)\right)$. We obtain

$$
\omega_{\alpha}\left(E\left(A_{0}^{\prime}\right)\right) \leq(1+\varepsilon) d_{i}\left|X_{i}\right| \cdot\left(d_{i}^{G} d_{0}+\hat{\varepsilon}\right) n \leq\left(d_{0} d_{i} d_{i}^{G}+2 \hat{\varepsilon}\right)\left|X_{i}\right| n
$$

Now (6.10) implies that $\omega_{\alpha}(M) \leq\left(1+2 \hat{\varepsilon}^{1 / 2}\right) d_{i}^{G} d_{i}\left|X_{i}\right|$, and hence $\left|E_{\alpha} \cap E\left(A_{i}^{*}\right)\right| \leq\left(1+\varepsilon^{\prime}\right) d_{i}^{G} d_{i}\left|X_{i}\right|$.

Now, consider $\alpha \in C_{G_{0 i}^{\prime}}$. Let $E_{\alpha} \subseteq E\left(G_{0 i}^{\prime}\right)$ be the set of edges of $G_{0 i}^{\prime}$ on which $\alpha$ appears. We define a weight function $\omega_{\alpha}: E\left(A_{0}^{\prime}\right) \rightarrow[\Lambda]_{0}$ by setting

$$
\omega_{\alpha}(x v):=\left|\left\{v_{i} \in N_{G_{0 i}^{\prime}}(v): v v_{i} \in E_{\alpha}, x x_{i} \in E\left(H\left[X_{0}, X_{i}\right]\right), x_{i} v_{i} \in E\left(A_{i}^{\prime}\right)\right\}\right|
$$

for every $x v \in E\left(A_{0}^{\prime}\right)$, and we add $\omega_{\alpha}$ to $\mathcal{W}$. Note that the number of edges of $A_{i}^{*}$ on which $\alpha$ appears is at most $\omega_{\alpha}(M)$.

To bound $\omega_{\alpha}(M)$, we again use (6.10) and seek an upper bound for $\omega_{\alpha}\left(E\left(A_{0}^{\prime}\right)\right)$. Since $c$ is $(1+\varepsilon) e_{G}\left(V_{0}, V_{i}\right) / e_{H}\left(X_{0}, X_{i}\right)$-bounded on $G\left[V_{0}, V_{i}\right]$ by assumption, we have $\left|E_{\alpha}\right| \leq\left(1+\varepsilon^{1 / 2}\right)$ $d_{i}^{G}\left|X_{i}\right| n / e_{H}\left(X_{0}, X_{i}\right)$.

For every edge $v v_{i} \in E_{\alpha}$ with $v_{i} \in V_{i} \backslash V_{i}^{b a d}$, condition (6.6) implies that

$$
e_{H}\left(N_{A_{0}^{\prime}}(v), N_{A_{i}^{\prime}}\left(v_{i}\right)\right)=\left(d_{0} d_{i} \pm \hat{\varepsilon}\right) e_{H}\left(X_{0}, X_{i}\right) .
$$

Hence, every edge $v v_{i} \in E_{\alpha}$ with $v_{i} \in V_{i} \backslash V_{i}^{\text {bad }}$ contributes weight $\left(d_{0} d_{i} \pm \hat{\varepsilon}\right) e_{H}\left(X_{0}, X_{i}\right)$ to $\omega_{\alpha}\left(E\left(A_{0}^{\prime}\right)\right)$. Since $\Delta\left(E_{\alpha}\right) \leq \Lambda$ and $\left|V_{i}^{\text {bad }}\right| \leq 3 r \varepsilon n$, there are at most $3 r \Lambda \varepsilon n$ edges $v v_{i} \in E_{\alpha}$ with $v_{i} \in V_{i}^{\text {bad }}$, each of which contributes weight at most $n$. We conclude that

$$
\begin{aligned}
\omega_{\alpha}\left(E\left(A_{0}^{\prime}\right)\right) & \leq \frac{\left(1+\varepsilon^{1 / 2}\right) d_{i}^{G}\left|X_{i}\right| n}{e_{H}\left(X_{0}, X_{i}\right)} \cdot\left(d_{0} d_{i}+\hat{\varepsilon}\right) e_{H}\left(X_{0}, X_{i}\right)+3 r \Lambda \varepsilon n^{2} \\
& \leq\left(d_{0} d_{i} d_{i}^{G}+2 \hat{\varepsilon}\right)\left|X_{i}\right| n
\end{aligned}
$$

Now (6.10) implies that $\omega_{\alpha}(M) \leq\left(1+\varepsilon^{\prime}\right) d_{i}^{G} d_{i}\left|X_{i}\right|$, completing the proof of (II) 6.3 .

Step 3.3. Checking (III) 6.3

Finally, we show that for all $i \in[r], \alpha \in C_{G_{0 i}^{\prime}}$, and $\beta \in C_{A_{i}^{\prime}}$, the pair $\{\alpha, \beta\}$ appears on at most $n^{\varepsilon}$ edges of $A_{i}^{*}$. This implies (III) 6.3 , as the codegree of a pair in $C_{A_{i}^{\prime}}$ is at most $K$ by assumption, and the codegree of a pair in $C_{G_{0 i}^{\prime}}$ is 0 . Fix $i \in[r], \alpha \in C_{G_{0 i}^{\prime}}$, and $\beta \in C_{A_{i}^{\prime}}$. Let

$$
E_{\alpha, \beta}:=\left\{v_{0} v_{i} x_{i}: v_{0} v_{i} \in E\left(G_{0 i}^{\prime}\right), x_{i} v_{i} \in E\left(A_{i}^{\prime}\right), c\left(v_{0} v_{i}\right)=\{\alpha\}, \beta \in c\left(x_{i} v_{i}\right)\right\}
$$

and define the weight function $\omega_{\alpha, \beta}: E\left(A_{0}^{\prime}\right) \rightarrow[\Lambda]_{0}$ by setting

$$
\omega_{\alpha, \beta}(x v):=\left|\left\{v v_{i} x_{i} \in E_{\alpha, \beta}: x x_{i} \in E\left(H^{+}\left[X_{0}, X_{i}\right]\right)\right\}\right| .
$$


Note that the number of edges of $A_{i}^{*}$ on which $\{\alpha, \beta\}$ appears is at most $\omega_{\alpha, \beta}(M)$. To bound $\omega_{\alpha, \beta}(M)$, note that every triple $v v_{i} x_{i} \in E_{\alpha, \beta}$ contributes weight at most 1 to $\omega_{\alpha, \beta}\left(E\left(A_{0}^{\prime}\right)\right)$. By assumption, $c$ is locally $\Lambda$-bounded and (globally) $(1+\varepsilon) d_{i}\left|X_{i}\right|$-bounded on $A_{i}$, which implies that $\omega_{\alpha, \beta}\left(E\left(A_{0}^{\prime}\right)\right) \leq$ $\left|E_{\alpha, \beta}\right| \leq(1+\varepsilon) d_{i} \Lambda\left|X_{i}\right| \leq 2 \Lambda n$. Now, (6.10) implies that $\omega_{\alpha, \beta}(M) \leq n^{\varepsilon}$. Hence, for all $i \in[r]$, $\alpha \in C_{G_{0 i}^{\prime}}$ and $\beta \in C_{A_{i}^{\prime}}$, we add the corresponding weight function $\omega_{\alpha, \beta}$ to $\mathcal{W}$, which implies (III) $)_{6.3}$. This completes the proof.

\section{Proof of Lemma 1.4}

In this section, we prove our rainbow blow-up lemma (Lemma 1.4). First, we will deduce Lemma 1.4 from a similar statement (Lemma 7.1), where we impose stronger conditions on $G$ and $H$. This reduction utilises the results of Section 5. We will conclude with the proof of Lemma 7.1.

Lemma 7.1. Let $1 / n \ll \varepsilon \ll \gamma, d, 1 / r, 1 / \Lambda$. Let $\left(H, G,\left(X_{i}\right)_{i \in[r]},\left(V_{i}\right)_{i \in[r]}\right)$ be an $(\varepsilon, d)$-super-regular blow-up instance. Assume further that

(i) $\left|V_{i}\right|=(1 \pm \varepsilon) n$ for all $i \in[r]$.

(ii) For all $i j \in\left(\begin{array}{c}{[r]} \\ 2\end{array}\right)$, the graph $H\left[X_{i}, X_{j}\right]$ is a matching of size at least $\gamma^{2} n$.

(iii) $c: E(G) \rightarrow C$ is a colour-split edge-colouring of $G$ such that $c$ is locally $\Lambda$-bounded and $c$ restricted to $G\left[V_{i}, V_{j}\right]$ is $(1-\gamma) e_{G}\left(V_{i}, V_{j}\right) / e_{H}\left(X_{i}, X_{j}\right)$-bounded for all $i j \in\left(\begin{array}{c}{[r]} \\ 2\end{array}\right)$.

Then there exists a rainbow embedding $\phi$ of $H$ into $G$ such that $\phi(x) \in V_{i}$ for all $i \in[r]$ and $x \in X_{i}$.

Proof of Lemma 1.4. We split the proof into three steps. In Step 1, we apply Lemma 5.2 to obtain a spanning subgraph $G_{1} \subseteq G$ such that the restricted edge-colouring is colour-split. In Step 2, we apply Lemma 5.4 to refine the partitions of $G_{1}$ and $H$ in such a way that the vertex classes of $H$ are 2-independent. Then, in Step 3, we can apply Lemma 7.1 to complete the proof.

In view of the statement, we may assume that

$$
1 / n \ll \varepsilon \ll \gamma \ll d, 1 / r, 1 / \Delta, 1 / \Lambda
$$

Choose new constants $\varepsilon_{1}, \varepsilon_{2}, \gamma^{\prime}, d_{1}, d_{2}$ with $\varepsilon \ll \varepsilon_{1} \ll \varepsilon_{2} \ll d_{2} \ll \gamma^{\prime} \ll d_{1} \ll \gamma$.

Step 1. Colour-splitting

First, let $H_{1}$ be a supergraph of $H$ on $V(H)$ such that $e_{H_{1}-H}\left(X_{i}, X_{j}\right) \leq \gamma^{2} n \leq e_{H_{1}}\left(X_{i}, X_{j}\right)$ for all $i j \in\left(\begin{array}{c}{[r]} \\ 2\end{array}\right)$ and $\Delta\left(H_{1}\right) \leq \Delta^{\prime}:=\Delta+r$. We claim that for all $\alpha \in C$, we have

$$
\sum_{i j \in\left(\begin{array}{c}
{[r]} \\
2
\end{array}\right)} e_{G}^{\alpha}\left(V_{i}, V_{j}\right) e_{H_{1}}\left(X_{i}, X_{j}\right) \leq\left(1-\frac{\gamma}{2}\right) d n^{2} .
$$

Indeed, since $c$ is locally $\Lambda$-bounded, we obtain that $e_{G}^{\alpha}\left(V_{i}, V_{j}\right) e_{H_{1}-H}\left(X_{i}, X_{j}\right) \leq 2 \Lambda n \cdot \gamma^{2} n \leq r^{-2} \cdot \gamma d n^{2} / 2$ for each $i j \in\left(\begin{array}{c}{[r]} \\ 2\end{array}\right)$. Hence, we can apply Lemma 5.2 to $\left(H_{1}, G,\left(X_{i}\right)_{i \in[r]},\left(V_{i}\right)_{i \in[r]}\right)$ (with $\gamma / 2, \Delta^{\prime}$ playing the roles of $\gamma, \Delta)$ and obtain a spanning subgraph $G_{1}$ of $G$ such that $\left(H_{1}, G_{1},\left(X_{i}\right)_{i \in[r]},\left(V_{i}\right)_{i \in[r]}\right)$ is an $\left(\varepsilon_{1}, d_{1}\right)$-super-regular blow-up instance and the colouring $c_{1}:=\left.c\right|_{E\left(G_{1}\right)}$ is colour-split, and

$$
\left(1-\frac{\gamma}{4}\right) \frac{e_{G_{1}}\left(V_{i}, V_{j}\right)}{e_{H_{1}}\left(X_{i}, X_{j}\right)} \text {-bounded }
$$

for each bipartite subgraph $G_{1}\left[V_{i}, V_{j}\right]$. Clearly, a rainbow embedding of $H_{1}$ into $G_{1}$ also yields a rainbow embedding of $H$ into $G$.

Step 2. Refining the vertex partitions 
We can now apply Lemma 5.4 to the $\left(\varepsilon_{1}, d_{1}\right)$-super-regular blow-up instance $\left(H_{1}, G_{1},\left(X_{i}\right)_{i \in[r]}\right.$, $\left.\left(V_{i}\right)_{i \in[r]}\right)$ with edge-colouring $c_{1}$ and $\gamma^{\prime}, \Delta^{\prime}$ playing the roles of $\gamma, \Delta$. Hence, we obtain an $\left(\varepsilon_{2}, d_{2}\right)$ super-regular blow-up instance

$$
\left(H_{2}, G_{2},\left(X_{i, j}\right)_{i \in[r], j \in\left[\Delta^{\prime 2}\right]},\left(V_{i, j}\right)_{i \in[r], j \in\left[\Delta^{\prime 2}\right]}\right)
$$

such that for $n^{\prime}:=n / \Delta^{\prime 2}$, we have that

(a) $\left(X_{i, j}\right)_{j \in\left[\Delta^{\prime 2}\right]}$ is partition of $X_{i}$ and $\left(V_{i, j}\right)_{j \in\left[\Delta^{\prime 2}\right]}$ is partition of $V_{i}$ for every $i \in[r]$, and $\left|X_{i, j}\right|=$ $\left|V_{i, j}\right|=\left(1 \pm \varepsilon_{2}\right) n^{\prime}$ for all $i \in[r], j \in\left[\Delta^{\prime 2}\right]$.

(b) $H_{2}$ is a supergraph of $H_{1}$ on $V(H)$ such that $H_{2}\left[X_{i_{1}, j_{1}}, X_{i_{2}, j_{2}}\right]$ is a matching of size at least $\gamma^{\prime 4} n^{\prime}$ for all $i_{1}, i_{2} \in[r], j_{1}, j_{2} \in\left[\Delta^{\prime 2}\right],\left(i_{1}, j_{1}\right) \neq\left(i_{2}, j_{2}\right)$.

(c) $G_{2}$ is a graph on $V(G)$ such that $G_{2}\left[V_{i_{1}, j_{1}}, V_{i_{2}, j_{2}}\right] \subseteq G_{1}\left[V_{i_{1}}, V_{i_{2}}\right]$ for all distinct $i_{1}, i_{2} \in[r]$ and all $j_{1}, j_{2} \in\left[\Delta^{\prime 2}\right]$.

(d) $c_{2}$ is an edge-colouring of $G_{2}$ such that $\left.c_{2}\right|_{E\left(G_{1}\right) \cap E\left(G_{2}\right)}=\left.c_{1}\right|_{E\left(G_{1}\right) \cap E\left(G_{2}\right)}$, and $c_{2}$ is colour-split with respect to the partition $\left(V_{i, j}\right)_{i \in[r], j \in\left[\Delta^{\prime 2}\right]}$, and $c_{2}$ is locally $\Lambda$-bounded, and $c_{2}$ restricted to $G_{2}\left[V_{i_{1}, j_{1}}, V_{i_{2}, j_{2}}\right]$ is

$$
\left(1-\frac{\gamma^{\prime}}{2}\right) \frac{e_{G_{2}}\left(V_{i_{1}, j_{1}}, V_{i_{2}, j_{2}}\right)}{e_{H_{2}}\left(X_{i_{1}, j_{1}}, X_{i_{2}, j_{2}}\right)} \text {-bounded }
$$

for all $i_{1}, i_{2} \in[r], j_{1}, j_{2} \in\left[\Delta^{\prime 2}\right],\left(i_{1}, j_{1}\right) \neq\left(i_{2}, j_{2}\right)$.

Again, a $c_{2}$-rainbow embedding of $H_{2}$ into $G_{2}$ also yields a $c_{1}$-rainbow embedding of $H_{1}$ into $G_{1}$.

\section{Step 3. Applying Lemma 7.1}

We can now complete the proof by applying Lemma 7.1 as follows:

\begin{tabular}{r||c|c|c|c|c|c} 
Parameter & $n^{\prime}$ & $\varepsilon_{2}$ & $\gamma^{\prime 2}$ & $d_{2}$ & $r \Delta^{\prime 2}$ & $\Lambda$ \\
\hline Plays the role of & $n$ & $\varepsilon$ & $\gamma$ & $d$ & $r$ & $\Lambda$
\end{tabular}

\begin{tabular}{r||c|c|c|c} 
Object & $H_{2}$ & $G_{2}$ & $\left(X_{i, j}\right)_{i \in[r], j \in\left[\Delta^{\prime 2}\right]}$ & $\left(V_{i, j}\right)_{i \in[r], j \in\left[\Delta^{\prime 2}\right]}$ \\
\hline Plays the role of & $H$ & $G$ & $\left(X_{i}\right)_{i \in[r]}$ & $\left(V_{i}\right)_{i \in[r]}$
\end{tabular}

This yields a rainbow embedding of $\mathrm{H}_{2}$ into $G_{2}$, and hence of $H$ in $G$.

We now deduce Theorem 1.3 from Lemma 1.4 by partitioning $H$ using the Hajnal-Szemerédi theorem (Theorem 5.3) and $G$ randomly.

Proof of Theorem 1.3. Let $r:=\Delta+1$. We may assume that $\varepsilon$ is sufficiently small and $n$ is sufficiently large. By applying Theorem 5.3 to $H$, we obtain a partition $\left(X_{i}\right)_{i \in[r]}$ of $V(H)$ into independent sets with $\left|X_{i}\right| \in\left\{\left\lfloor\frac{n}{r}\right\rfloor,\left\lceil\frac{n}{r}\right\rceil\right\}$. We claim that there exists a partition $\left(V_{i}\right)_{i \in[r]}$ of $V(G)$ such that

(i) $G\left[V_{i}, V_{j}\right]$ is $(2 r \varepsilon, d)$-super-regular for all $i j \in\left(\begin{array}{c}{[r]} \\ 2\end{array}\right)$.

(ii) For all $\alpha \in C$ with $e^{\alpha}(G) \geq n^{3 / 4}$, we have $e_{G}^{\alpha}\left(V_{i}, V_{j}\right)=(1 \pm \varepsilon) 2 e^{\alpha}(G) / r^{2}$ for all $i j \in\left(\begin{array}{c}{[r]} \\ 2\end{array}\right)$.

(iii) $\left|V_{i}\right|=\left|X_{i}\right|$ for all $i \in[r]$.

That such a partition exists can be seen using a probabilistic argument: For each $v \in V(G)$ independently, choose a label $i \in[r]$ uniformly at random, and put $v$ into $V_{i}$. Using Chernoff's inequality (Theorem 4.1) for (i) and McDiarmid's inequality (Theorem 4.2) for (ii), it is easy to check that (i) and (ii) are satisfied with probability at least $1-\mathrm{e}^{-n^{1 / 3}}$. Moreover, (iii) holds with probability $\Omega\left(n^{-r / 2}\right)$. Hence, such a partition exists.

Therefore, we conclude that $\left(H, G,\left(X_{i}\right)_{i \in[r]},\left(V_{i}\right)_{i \in[r]}\right)$ is a $(2 r \varepsilon, d)$-super-regular blow-up instance. Consider $\alpha \in C$. If $e^{\alpha}(G) \leq n^{3 / 4}$, then condition (iii) in Lemma 1.4 clearly holds. If $e^{\alpha}(G) \geq n^{3 / 4}$, we 
use (ii) to see that

$$
\begin{aligned}
\sum_{i j \in\left(\begin{array}{c}
{[r]} \\
2
\end{array}\right)} e_{G}^{\alpha}\left(V_{i}, V_{j}\right) e_{H}\left(X_{i}, X_{j}\right) & =(1 \pm \varepsilon) 2 e^{\alpha}(G) e(H) / r^{2} \leq(1+\varepsilon)(1-\gamma) 2 e(G) / r^{2} \\
& \leq(1-\gamma / 2) d(n / r)^{2}
\end{aligned}
$$

Thus, we can apply Lemma 1.4 and obtain a rainbow copy of $H$ in $G$.

It remains to prove Lemma 7.1. The proof splits into four steps as follows. In Step 1, we split $G$ into two spanning subgraphs $G_{A}$ and $G_{B}$ with disjoint colour sets. In Step 2, we define the necessary 'candidacy graphs' that we track during the approximate embedding in Step 3. We then iteratively apply Lemma 6.3 in Step 3 to find approximate rainbow embeddings of $X_{i}$ into $V_{i}$ using only the edges of $G_{A}$. All those steps have to be performed carefully such that we can employ Lemma 4.7 in Step 4 and use the reserved set of colours of $G_{B}$ to turn the approximate rainbow embedding into a complete one.

Proof of Lemma 7.1. In view of the statement, we may assume that $\gamma \ll d, 1 / r, 1 / \Lambda$. Choose new constants $\varepsilon_{0}, \varepsilon_{1}, \ldots, \varepsilon_{r+1}, \mu$ with $\varepsilon \ll \varepsilon_{0} \ll \varepsilon_{1} \ll \cdots \ll \varepsilon_{r+1} \ll \mu \ll \gamma$. For $i \in[r]$, let

$$
x_{i}:=\bigcup_{j \in[i]} X_{j}, \quad V_{i}:=\bigcup_{j \in[i]} V_{j}
$$

\section{Step 1. Colour splitting}

To reserve an exclusive set of colours for the application of Lemma 4.7, we randomly partition the edges of $G$ into two spanning subgraphs $G_{A}$ and $G_{B}$ as follows. For each colour class of $G$ independently, we add its edges to $G_{A}$ with probability $1-\gamma$ and otherwise to $G_{B}$. Let $d_{A}:=(1-\gamma) d$ and $d_{B}:=\gamma d$. By Lemma 5.1, we conclude that with probability at least $1-1 / n$,

$$
G_{Z}\left[V_{i}, V_{j}\right] \text { is }\left(\varepsilon_{0}^{2}, d_{Z}\right) \text {-super-regular for all } i j \in\left(\begin{array}{c}
{[r]} \\
2
\end{array}\right), Z \in\{A, B\} \text {. }
$$

Hence, we may assume that $G$ is partitioned into $G_{A}$ and $G_{B}$ such that (7.1) holds.

Step 2. Candidacy graphs

We want to show that there is a partial rainbow embedding of $H\left[\mathcal{X}_{r}\right]$ into $G_{A}\left[\mathcal{V}_{r}\right]$ that maps almost all vertices of $X_{i}$ into $V_{i}$ for every $i \in[r]$. Moreover, we need to ensure certain conditions for the remaining unembedded vertices in order to finally apply Lemma 4.7. We will achieve this by iteratively applying the Approximate Embedding Lemma (Lemma 6.3) in Step 3. To formally state the induction hypothesis, we need some preliminary definitions.

For $t \in[r]_{0}$, we call $\phi_{t}: X_{1}^{\phi_{t}} \cup \ldots \cup X_{t}^{\phi_{t}} \rightarrow V_{1}^{\phi_{t}} \cup \ldots \cup V_{t}^{\phi_{t}}$ a $t$-partial embedding if $X_{i}^{\phi_{t}} \subseteq X_{i}$, $V_{i}^{\phi_{t}} \subseteq V_{i}$, and $\phi_{t}\left(X_{i}^{\phi_{t}}\right)=V_{i}^{\phi_{t}}$ for every $i \in[t]$, such that $\phi_{t}$ is an embedding of $H\left[X_{1}^{\phi_{t}} \cup \ldots \cup X_{t}^{\phi_{t}}\right]$ into $G_{A}\left[V_{1}^{\phi_{t}} \cup \ldots \cup V_{t}^{\phi_{t}}\right]$. For brevity, define

$$
X_{t}^{\phi_{t}}:=\bigcup_{i \in[t]} X_{i}^{\phi_{t}}, \quad \mathcal{V}_{t}^{\phi_{t}}:=\bigcup_{i \in[t]} V_{i}^{\phi_{t}} .
$$

Given a $t$-partial embedding $\phi_{t}$, we define two kinds of bipartite auxiliary graphs: for each $i \in[r] \backslash[t]$, we define a graph $A_{i}\left(\phi_{t}\right)$ with bipartition $\left(X_{i}, V_{i}\right)$ that tracks the still-available images of a vertex $x \in X_{i}$ in $G_{A}$, which will be used to extend the $t$-partial rainbow embedding $\phi_{t}$ to a $(t+1)$-partial rainbow embedding $\phi_{t+1}$ via Lemma 6.3 in Step 3. Moreover, for each $i \in[r]$, we define a bipartite graph $B_{i}\left(\phi_{t}\right)$ that tracks the potential images of a vertex $x \in X_{i}$ in $G_{B}$, which will be used for the completion via Lemma 4.7 in Step 4. Here, we keep tracking potential images of vertices even if they have been embedded, since in Step 4, we will actually 'unembed' a few vertices.

When extending $\phi_{t}$ to $\phi_{t+1}$, we intend to update the graphs $A_{i}\left(\phi_{t}\right)$ and $B_{i}\left(\phi_{t}\right)$ simultaneously using Lemma 6.3. To facilitate this, we define $B_{i}\left(\phi_{t}\right)$ on a copy $\left(X_{i}^{B}, V_{i}^{B}\right)$ of the bipartition $\left(X_{i}, V_{i}\right)$. For every 
$i \in[r]$, let $X_{i}^{B}$ and $V_{i}^{B}$ be disjoint copies of $X_{i}$ and $V_{i}$, respectively. Let $\pi$ be the bijection that maps a vertex in $\bigcup_{i \in[r]}\left(X_{i} \cup V_{i}\right)$ to its copy in $\bigcup_{i \in[r]}\left(X_{i}^{B} \cup V_{i}^{B}\right)$. Let $G^{+}$and $H^{+}$be supergraphs of $G_{A}$ and $H$ with vertex partitions $\left(V_{1}, \ldots, V_{r}, V_{1}^{B}, \ldots, V_{r}^{B}\right)$ and $\left(X_{1}, \ldots, X_{r}, X_{1}^{B}, \ldots, X_{r}^{B}\right)$, respectively, and edge sets

$$
\begin{aligned}
& E\left(G^{+}\right):=E\left(G_{A}\right) \cup\left\{u \pi(v), v \pi(u): u v \in E\left(G_{B}\right)\right\} \cup E_{G}^{*}, \\
& E\left(H^{+}\right):=E(H) \cup\{x \pi(y), y \pi(x): x y \in E(H)\} \cup E_{H}^{*},
\end{aligned}
$$

where we added for convenience a suitable set $E_{G}^{*} \subseteq \bigcup_{i \in[r]}\left\{u v: u \in V_{i}, v \in V_{i}^{B}\right\}$ such that $G^{+}\left[V_{i}, V_{i}^{B}\right]$ is $\left(\varepsilon_{0}, d_{B}\right)$-super-regular for all $i \in[r]$, and the set $E_{H}^{*}:=\{x \pi(x): x \in V(H)\}$ so that $H^{+}\left[X_{i}, X_{i}^{B}\right]$ is a perfect matching for all $i \in[r]$. Note that $G^{+}\left[V_{i}, V_{j}\right]=G_{A}\left[V_{i}, V_{j}\right]$, whereas $G^{+}\left[V_{i}, V_{j}^{B}\right]$ and $G^{+}\left[V_{i}^{B}, V_{j}\right]$ are isomorphic to $G_{B}\left[V_{i}, V_{j}\right]$ for all $i j \in\left(\begin{array}{c}{[r]} \\ 2\end{array}\right)$.

We now define $A_{i}\left(\phi_{t}\right)$ and $B_{i}\left(\phi_{t}\right)$. Let $X_{i}^{A}:=X_{i}$ and $V_{i}^{A}:=V_{i}$ for every $i \in[r]$. For $Z \in\{A, B\}$ and $i \in[r]$, we say that $v_{i} \in V_{i}^{Z}$ is a candidate for $x_{i} \in X_{i}^{Z}$ (given $\phi_{t}$ ) if

$$
\phi_{t}\left(N_{H^{+}}\left(x_{i}\right) \cap X_{t}^{\phi_{t}}\right) \subseteq N_{G^{+}}\left(v_{i}\right),
$$

and we define $Z_{i}\left(\phi_{t}\right)$ as the bipartite graph with partition $\left(X_{i}^{Z}, V_{i}^{Z}\right)$ and edge set

$$
E\left(Z_{i}\left(\phi_{t}\right)\right):=\left\{x_{i} v_{i}: x_{i} \in X_{i}^{Z}, v_{i} \in V_{i}^{Z} \text {, and } v_{i} \text { is a candidate for } x_{i} \text { given } \phi_{t}\right\} .
$$

We call any spanning subgraph of $Z_{i}\left(\phi_{t}\right)$ a candidacy graph.

Next, we define edge set colourings for these candidacy graphs. For $i \in[r] \backslash[t]$, we assign to every edge $e=x_{i} v_{i} \in E\left(A_{i}\left(\phi_{t}\right)\right)$ a colour set $c_{t}(e)$ of size at most $t$, which represents the colours that would be used if we were to embed $x_{i}$ at $v_{i}$ in the next step. More precisely, for every $i \in[r] \backslash[t]$ and every edge $x_{i} v_{i} \in E\left(A_{i}\left(\phi_{t}\right)\right)$, we set

$$
c_{t}\left(x_{i} v_{i}\right):=c\left(E\left(G_{A}\left[\phi_{t}\left(N_{H}\left(x_{i}\right) \cap X_{t}^{\phi_{t}}\right),\left\{v_{i}\right\}\right]\right)\right) .
$$

Tracking this set will help us to ensure that the embedding is rainbow when we extend $\phi_{t}$ to $\phi_{t+1}$. Since $\left|N_{H}\left(x_{i}\right) \cap X_{t}^{\phi_{t}}\right| \leq t$ and $|c(e)|=1$ for all $e \in E\left(G_{A}\right)$, we have $\left|c_{t}\left(x_{i} v_{i}\right)\right| \leq t$.

For the candidacy graphs $B_{i}\left(\phi_{t}\right)$, we merely need to know that they maintain super-regularity during the inductive approximate embedding (see $\mathbf{S}(\mathrm{t})$ below). Hence, for convenience, we set $c_{t}(e):=\emptyset$ for every $e \in E\left(B_{i}\left(\phi_{t}\right)\right)$.

We also assign artificial dummy colours to the edges of $E\left(G^{+}\right) \backslash E\left(G_{A}\right)$ as follows. Let $c^{\text {art }}:\left(\begin{array}{c}V\left(G^{+}\right) \\ 2\end{array}\right) \rightarrow C^{\text {art }}$ be a rainbow edge-colouring of all possible edges in $V\left(G^{+}\right)$such that $C^{\text {art }} \cap C^{2}=\emptyset$. Define $c^{+}$on $E\left(G^{+}\right)$by setting $c^{+}(e):=c(e)$ if $e \in E\left(G_{A}\right)$ and $c^{+}(e):=c^{\text {art }}(e)$ otherwise.

\section{Step 3. Induction}

We inductively prove the following statement $\mathbf{S}(\mathrm{t})$ for all $t \in[r]_{0}$.

$\mathbf{S}(\mathrm{t})$. There exists a $t$-partial rainbow embedding $\phi_{t}: X_{t}^{\phi_{t}} \rightarrow V_{t}^{\phi_{t}}$ with $\left|X_{s}^{\phi_{t}}\right|=\left|V_{s}^{\phi_{t}}\right| \geq\left(1-\varepsilon_{t}\right)\left|X_{s}\right|$ for all $s \in[t]$; and for all $Z \in\{A, B\}$, there exists a candidacy graph $Z_{i}^{t} \subseteq Z_{i}\left(\phi_{t}\right)$ such that

(a) $A_{i}^{t}$ is $\left(\varepsilon_{t}, d_{A}^{t}\right)$-super-regular for all $i \in[r] \backslash[t]$.

(b) $B_{i}^{t}$ is $\left(\varepsilon_{t}, d_{B}^{t}\right)$-super-regular for all $i \in[r]$.

(c) The colouring $c_{t}$ restricted to $A_{i}^{t}$ is $\left(1+\varepsilon_{t}\right) d_{A}^{t}\left|X_{i}\right|$-bounded and has codegree at most $n^{1 / 3}$ for all $i \in[r] \backslash[t]$.

The statement $\mathbf{S}(0)$ holds for $\phi_{0}$ being the empty function: clearly, for all $Z \in\{A, B\}, i \in[r]$, the candidacy graph $Z_{i}\left(\phi_{0}\right)$ is complete bipartite, and by (7.3), we have $c_{0}(e)=\emptyset$ for all $e \in E\left(A_{i}\left(\phi_{0}\right)\right)$, implying $\mathbf{S}(0)$. 
Hence, we may assume the truth of $\mathbf{S}(\mathrm{t})$ for some $t \in[r-1]_{0}$ and let $\phi_{t}: X_{t}^{\phi_{t}} \rightarrow \mathcal{V}_{t}^{\phi_{t}}$ and $A_{i}^{t}$, $B_{i}^{t}$ be as in $\mathbf{S}(\mathrm{t})$. We will now extend $\phi_{t}$ to a $(t+1)$-partial rainbow embedding $\phi_{t+1}$ such that $\mathbf{S}(\mathrm{t}+1)$ holds. Note that any matching $\sigma: X_{t+1}^{\sigma} \rightarrow V_{t+1}^{\sigma}$ in $A_{t+1}^{t}$ with $X_{t+1}^{\sigma} \subseteq X_{t+1}$ and $V_{t+1}^{\sigma} \subseteq V_{t+1}$ induces an embedding $\phi_{t+1}: X_{t}^{\phi_{t}} \cup X_{t+1}^{\sigma} \rightarrow \mathcal{V}_{t}^{\phi_{t}} \cup V_{t+1}^{\sigma}$, which extends $\phi_{t}$ to a $(t+1)$-partial embedding as follows:

$$
\phi_{t+1}(x):= \begin{cases}\phi_{t}(x) & \text { if } x \in X_{t}^{\phi_{t}} \\ \sigma(x) & \text { if } x \in X_{t+1}^{\sigma}\end{cases}
$$

The following is a key observation: since $c$ is colour-split and by definition of the candidacy graph $A_{t+1}^{t}$ and the colouring $c_{t}$ on $E\left(A_{t+1}^{t}\right)$, whenever $\sigma$ is a rainbow matching in $A_{t+1}^{t}$, then $\phi_{t+1}$ is a $(t+1)$-partial rainbow embedding.

Now, we aim to apply Lemma 6.3 to obtain an almost perfect rainbow matching $\sigma$ in $A_{t+1}^{t}$. Let $H^{t+1}:=H^{+}-X_{t}$, and let $G^{t+1}:=G^{+}-\mathcal{V}_{t}$. We claim that

$$
\left(H^{t+1}, G^{t+1}, \mathcal{A}, c^{+} \cup c_{t}\right) \text { is an }\left(\varepsilon_{t}, \boldsymbol{d},\left(d_{A}^{t}, \boldsymbol{d}^{t}\right), t, \Lambda\right) \text {-embedding-instance, }
$$

where $\mathcal{A}:=\left(A_{t+1}^{t}, \ldots, A_{r}^{t}, B_{1}^{t}, \ldots, B_{r}^{t}\right)$ and $\mathbf{d}:=\left(d_{A}, \ldots, d_{A}, d_{B}, \ldots, d_{B}\right)\left(d_{A}\right.$ repeated $r-t-1$ times and $d_{B}$ repeated $r$ times).

First, note that the colouring $c^{+} \cup c_{t}$ is locally $\Lambda$-bounded and colour-split with respect to the vertex partition

$$
\left(X_{t+1}, \ldots, X_{r}, X_{1}^{B}, \ldots, X_{r}^{B}, V_{t+1}, \ldots, V_{r}, V_{1}^{B}, \ldots, V_{r}^{B}\right)
$$

of $G^{t+1} \cup \bigcup_{i \in[r] \backslash[t]} A_{i}^{t} \cup \bigcup_{i \in[r]} B_{i}^{t}$. Moreover, the colour sets of $G^{t+1}$-edges have size 1, and the colour sets of candidacy graph edges have size at most $t$.

Further, the super-regularity of the $G^{t+1}$-pairs follows from (7.1) (and for the pair $G^{t+1}\left[V_{t+1}, V_{t+1}^{B}\right]$ from the choice of $E_{G}^{*}$ ). Moreover, combining (7.1) with assumption (iii), we infer that for every $i \in[r-t-1]$, the edge-colouring

$$
c \text { restricted to } G_{A}\left[V_{t+1}, V_{t+1+i}\right] \text { is }\left(1+\varepsilon_{t}\right) \frac{e_{G_{A}}\left(V_{t+1}, V_{t+1+i}\right)}{e_{H}\left(X_{t+1}, X_{t+1+i}\right)} \text {-bounded. }
$$

Finally, the super-regularity of the candidacy graphs and the boundedness of their colourings follows from $\mathbf{S}(\mathrm{t})$. We conclude that (7.5) holds. Hence, we can apply Lemma 6.3 to this instance with the following parameters:

\begin{tabular}{rl||c|c|c|c|c|c} 
Parameter & $\left|X_{t+1}\right|$ & $\varepsilon_{t}$ & $\varepsilon_{t+1}$ & $t$ & $r-t-1+r$ & $\Lambda$ \\
\hline Plays the role of & $n$ & $\varepsilon$ & $\varepsilon^{\prime}$ & $t$ & $r$ & $\Lambda$ \\
& $n^{1 / 3}$ & $\mathbf{d}$ & $\left(d_{A}^{t}, \mathbf{d}^{t}\right)$ & & \\
\hline & $K$ & $\left(d_{i}^{G}\right)_{i \in[r]}$ & $\left(d_{i}\right)_{i \in[r]_{0}}$ &
\end{tabular}

Let $\sigma: X_{t+1}^{\sigma} \rightarrow V_{t+1}^{\sigma}$ be the rainbow matching in $A_{t+1}^{t}$ obtained from Lemma 6.3 with $\left|X_{t+1}^{\sigma}\right| \geq$ $\left(1-\varepsilon_{t+1}\right)\left|X_{t+1}\right|$. The matching $\sigma$ extends $\phi_{t}$ to a $(t+1)$-partial rainbow embedding $\phi_{t+1}$, as defined in (7.4). By Definition 6.1, the updated candidacy graphs with respect to $\sigma$ obtained from Lemma 6.3 are also updated candidacy graphs with respect to $\phi_{t+1}$ as defined in Step 2. (More precisely, we have $Z_{i}^{t, \sigma} \subseteq Z_{i}\left(\phi_{t+1}\right)$ for $Z \in\{A, B\}$.) Hence, by Lemma 6.3, we obtain new candidacy graphs $A_{i}^{t+1} \subseteq A_{i}\left(\phi_{t+1}\right)$ for $i \in[r] \backslash[t+1]$ and $B_{i}^{t+1} \subseteq B_{i}\left(\phi_{t+1}\right)$ for $i \in[r]$ that satisfy (I) $6.3-(\mathrm{III})_{6.3}$. By (I) 6.3 , we know that $A_{i}^{t+1}$ is $\left(\varepsilon_{t+1}, d_{A}^{t+1}\right)$-super-regular for every $i \in[r] \backslash[t+1]$, and $B_{i}^{t+1}$ is $\left(\varepsilon_{t+1}, d_{B}^{t+1}\right)$ super-regular for every $i \in[r]$, which implies $\mathbf{S}(\mathrm{t}+1)$ (a) and $\mathbf{S}(\mathrm{t}+1)(\mathrm{b})$. Moreover, the new colouring $c_{t+1}$, as defined in (7.3), corresponds to the updated colouring as in Definition 6.2, so we can assume that $c_{t+1}$ satisfies (II) 6.3 and (III) 6.3 . Thus, for every $i \in[r] \backslash[t+1]$, the colouring $c_{t+1}$ restricted to $A_{i}^{t+1}$ 
is $\left(1+\varepsilon_{t+1}\right) d_{A}^{t+1}\left|X_{i}\right|$-bounded by (II) 6.3 and has codegree at most $n^{1 / 3}$ by (III) 6.3 . This implies $\mathbf{S}(\mathrm{t}+1)(\mathrm{c})$ and hence completes the inductive step.

\section{Step 4. Completion}

We may assume that $\phi_{r}: X_{r}^{\phi_{r}} \rightarrow \mathcal{V}_{r}^{\phi_{r}}$ is an $r$-partial embedding fulfilling $\mathbf{S}(\mathrm{r})$ with $\left(\varepsilon_{r}, d_{B}^{r}\right)$-superregular candidacy graphs $B_{i}^{r} \subseteq B_{i}\left(\phi_{r}\right)$. Recall that we defined the bipartite candidacy graphs $B_{i}^{r}$ on copies $\left(X_{i}^{B}, V_{i}^{B}\right)$ only to conveniently apply Lemma 6.3 in Step 3. We now identify $B_{i}^{r}$ with a bipartite graph $B_{i}^{\prime}$ on $\left(X_{i}, V_{i}\right)$ and edge set $E\left(B_{i}^{\prime}\right):=\left\{x_{i} v_{i}: \pi\left(x_{i}\right) \pi\left(v_{i}\right) \in E\left(B_{i}^{r}\right)\right\}$. Hence, for each $i \in[r], B_{i}^{\prime}$ is $\left(\varepsilon_{r}, d_{B}^{r}\right)$-super-regular; and for every edge $x_{i} v_{i} \in E\left(B_{i}^{\prime}\right)$, we deduce from (7.2) that

$$
\phi_{r}\left(N_{H}\left(x_{i}\right) \cap X_{r}^{\phi_{r}}\right) \subseteq N_{G_{B}}\left(v_{i}\right)
$$

We want to apply Lemma 4.7 in order to complete the embedding using the edges in $G_{B}$ and the candidacy graphs $\left(B_{i}^{\prime}\right)_{i \in[r]}$. For every $i \in[r]$, let $\bar{V}_{i}:=V_{i} \backslash V_{i}^{\phi_{r}}$ and $\bar{X}_{i}:=X_{i} \backslash X_{i}^{\phi_{r}}$ be the sets of unused/unembedded vertices. Note that we have no control over these sets except knowing that they are very small. To be able to apply Lemma 4.7, we now (randomly) add vertices that have already been embedded back to the unembedded vertices. That is, we will find sets $V_{i}^{\prime} \supseteq \bar{V}_{i}$ and $X_{i}^{\prime} \supseteq \bar{X}_{i}$ of size exactly $n_{B}:=\lceil\mu n\rceil$ (same size required for condition (ii) in Lemma 4.7) such that $B_{i}^{\prime}\left[X_{i}^{\prime}, V_{i}^{\prime}\right]$ is still super-regular.

For the application of Lemma 4.7, we also have to ensure not only that the colouring $c$ restricted to $G_{B}\left[V_{1}^{\prime} \cup \ldots \cup V_{r}^{\prime}\right]$ is sufficiently bounded (see property (c) below) but also that the colouring $c$ restricted to $G_{B}$ between already embedded sets $V_{i} \backslash V_{i}^{\prime}$ and sets $V_{j}^{\prime}$ used for the completion is sufficiently bounded (see property (d) below). Therefore, for $i, j \in[r]$, let $G_{B}^{\text {hit }}\left[V_{i} \backslash V_{i}^{\prime}, V_{j}^{\prime}\right]$ be the spanning subgraph of $G_{B}\left[V_{i} \backslash V_{i}^{\prime}, V_{j}^{\prime}\right]$ containing those edges $v_{i} v_{j} \in E\left(G_{B}\left[V_{i} \backslash V_{i}^{\prime}, V_{j}^{\prime}\right]\right)$ for which $\phi_{r}^{-1}\left(v_{i}\right)$ has an $H$-neighbour in $X_{j}^{\prime}$. That is, $G_{B}^{h i t}\left[V_{i} \backslash V_{i}^{\prime}, V_{j}^{\prime}\right]$ contains all the edges between $V_{i} \backslash V_{i}^{\prime}$ and $V_{j}^{\prime}$ that will potentially be used to extend the partial embedding when applying Lemma 4.7.

We claim that sets $\bar{V}_{i}^{+} \subseteq V_{i}^{\phi_{r}}$ can be chosen such that, setting $\bar{X}_{i}^{+}:=\phi_{r}^{-1}\left(\bar{V}_{i}^{+}\right), V_{i}^{\prime}:=\bar{V}_{i} \cup \bar{V}_{i}^{+}$, and $X_{i}^{\prime}:=\bar{X}_{i} \cup \bar{X}_{i}^{+}$, we have:

(a) $G_{B}\left[V_{i}^{\prime}, V_{j}^{\prime}\right]$ is $\left(\varepsilon_{r+1}, d_{B}\right)$-super-regular for all $i j \in\left(\begin{array}{c}{[r]} \\ 2\end{array}\right)$.

(b) $B_{i}^{\prime}\left[X_{i}^{\prime}, V_{i}^{\prime}\right]$ is $\left(\varepsilon_{r+1}, d_{B}^{r}\right)$-super-regular for every $i \in[r]$.

(c) The colouring $c$ restricted to $G_{B}\left[V_{1}^{\prime} \cup \ldots \cup V_{r}^{\prime}\right]$ is $\mu^{3 / 2} n$-bounded.

(d) The colouring $c$ restricted to $G_{B}^{h i t}\left[V_{i} \backslash V_{i}^{\prime}, V_{j}^{\prime}\right]$ is $\mu^{3 / 2} n$-bounded for all $i, j \in[r]$.

(e) $\left|V_{i}^{\prime}\right|=\left|X_{i}^{\prime}\right|=n_{B}$ for every $i \in[r]$.

This can be seen with a probabilistic argument. Independently for every $i \in[r]$ and $v \in V_{i}^{\phi_{r}}$, let $v$ belong to $\bar{V}_{i}^{+}$with probability $p_{i}:=\left(n_{B}-\left|\bar{V}_{i}\right|\right) /\left|V_{i}^{\phi_{r}}\right|$. We now show that (a)-(e) hold simultaneously with positive probability.

Note that $p_{i}=\mu \pm \sqrt{\varepsilon_{r}}$. Recall that $G_{B}\left[V_{i}, V_{j}\right]$ is $\left(\varepsilon_{0}, d_{B}\right)$-super-regular, $B_{i}^{\prime}$ is $\left(\varepsilon_{r}, d_{B}^{r}\right)$-super-regular, $\left|\bar{V}_{i}\right|=\left|\bar{X}_{i}\right| \leq 2 \varepsilon_{r} n$, and $c$ is locally $\Lambda$-bounded. Using Chernoff's bound, it is routine to show that (a) and (b) hold with probability at least $1-\mathrm{e}^{-\sqrt{n}}$, say. Note here that the regularity follows easily from the regularity of the respective supergraphs.

We show next that (d) also holds with high probability. Let $i, j \in[r]$, and let $\alpha$ be a colour. Let $X$ be the number of $\alpha$-coloured edges $v_{i} v_{j}$ in $\left.G_{B}\left[V_{i} \backslash V_{i}^{\prime}, V_{j}^{\prime}\right]\right)$ for which $v_{j} \in \bar{V}_{j}^{+}$and $\phi_{r}^{-1}\left(v_{i}\right)$ has an $H$-neighbour in $\bar{X}_{j}^{+}$. Note that since $\left|\bar{V}_{j}\right|=\left|\bar{X}_{j}\right| \leq 2 \varepsilon_{r} n$ and $c$ is locally $\Lambda$-bounded, the number of $\alpha$-coloured edges $v_{i} v_{j}$ in $\left.G_{B}\left[V_{i} \backslash V_{i}^{\prime}, V_{j}^{\prime}\right]\right)$ for which $v_{j} \in \bar{V}_{j}$ or $\phi_{r}^{-1}\left(v_{i}\right)$ has an $H$-neighbour in $\bar{X}_{j}$, is at most $4 \Lambda \varepsilon_{r} n$. Now, consider an edge $v_{i} v_{j} \in E\left(G_{B}\left[V_{i}^{\phi_{r}}, V_{j}^{\phi_{r}}\right]\right)$ with $\left\{x_{j}\right\}=N_{H}\left(\phi_{r}^{-1}\left(v_{i}\right)\right) \cap X_{j}^{\phi_{r}}$. Crucially, observe that $x_{j} \neq \phi_{r}^{-1}\left(v_{j}\right)$ because $v_{i} v_{j}$ is an edge in $G_{B}$ and therefore not in $G_{A}$. This 
implies that

$$
\mathbb{P}\left[v_{j} \in \bar{V}_{j}^{+}, x_{j} \in \bar{X}_{j}^{+}\right]=p_{j}^{2} \leq 2 \mu^{2} .
$$

Since $c$ is locally $\Lambda$-bounded, $\alpha$ appears on at most $2 \Lambda n$ such edges $v_{i} v_{j}$, and hence

$$
\mathbb{E}[X] \leq 4 \Lambda \mu^{2} n
$$

Since $c$ is locally $\Lambda$-bounded, an application of McDiarmid's inequality yields that, with probability at least $1-\mathrm{e}^{-n^{2 / 3}}$, we have $X \leq 5 \Lambda \mu^{2} n$, which implies that the number of $\alpha$-coloured edges in $G_{B}^{h i t}\left[V_{i} \backslash V_{i}^{\prime}, V_{j}^{\prime}\right]$ is at most $\mu^{3 / 2} n$. Together with a union bound, we infer that (d) holds with probability at least $1-\mathrm{e}^{-\sqrt{n}}$.

A similar (even simpler) argument using the local boundedness of $c$ and McDiarmid's inequality also works for (c). Thus, a union bound implies that (a)-(d) hold simultaneously with probability at least $1-4 \mathrm{e}^{-\sqrt{n}}$. Moreover, standard properties of the binomial distribution yield that $\left|\bar{V}_{i}^{+}\right|=n_{B}-\left|\bar{V}_{i}\right|$ (and thus, $\left.\left|V_{i}^{\prime}\right|=\left|X_{i}^{\prime}\right|=n_{B}\right)$ for all $i \in[r]$ with probability at least $\Omega\left(n^{-r / 2}\right)$. Hence, there exist such sets $X_{i}^{\prime}$ and $V_{i}^{\prime}$ for all $i \in[r]$ satisfying (a)-(e).

Let

$$
\begin{array}{ll}
X_{r}^{\prime}:=\bigcup_{i \in[r]} X_{i}^{\prime}, & V_{r}^{\prime}:=\bigcup_{i \in[r]} V_{i}^{\prime}, \\
X_{0}^{\prime}:=X_{r} \backslash X_{r}^{\prime}, & V_{0}^{\prime}:=\mathcal{V}_{r} \backslash \mathcal{V}_{r}^{\prime} .
\end{array}
$$

The restriction of $\phi_{r}$ to $X_{0}^{\prime}$ clearly yields a rainbow embedding $\psi_{0}: X_{0}^{\prime} \rightarrow V_{0}^{\prime}$ of $H\left[X_{0}^{\prime}\right]$ into $G_{A}\left[V_{0}^{\prime}\right]$. Let $G^{\prime}:=G_{B}\left[\mathcal{V}_{r}^{\prime}\right] \cup G_{B}^{\text {hit }}\left[V_{0}^{\prime}, \mathcal{V}_{r}^{\prime}\right]$, and let $H^{\prime}$ be the subgraph of $H$ with partition $\left(X_{i}^{\prime}\right)_{i \in[r]_{0}}$ that arises from $H$ by discarding all edges in $H\left[X_{0}^{\prime}\right]$. (This is feasible since edges within $X_{0}^{\prime}$ have already been embedded by $\left.\psi_{0}.\right)$ By (a) and (b), we have that $\mathcal{B}^{\prime}:=\left(H^{\prime}, G^{\prime},\left(X_{i}^{\prime}\right)_{i \in[r]_{0}},\left(V_{i}^{\prime}\right)_{i \in[r]_{0}}\right)$ is an $\left(\varepsilon_{r+1}, d_{B}\right)$ super-regular blow-up instance with exceptional sets $\left(X_{0}^{\prime}, V_{0}^{\prime}\right)$ and $\left(\varepsilon_{r+1}, d_{B}^{r}\right)$-super-regular candidacy graphs $\left(B_{i}^{\prime}\left[X_{i}^{\prime}, V_{i}^{\prime}\right]\right)_{i \in[r]}$. Moreover, $c$ restricted to $G^{\prime}$ is $\mu^{1 / 2} n_{B}$-bounded by (c) and (d), and all clusters have the same size $n_{B}$ by (e). Further,

○ From (7.6) and the definition of $G_{B}^{\text {hit }}$, it holds that for all $x \in X_{0}^{\prime}, i \in[r]$ and $x_{i} \in N_{H^{\prime}}(x) \cap X_{i}^{\prime}$, we have $N_{B_{i}^{\prime}}\left(x_{i}\right) \subseteq N_{G^{\prime}}\left(\psi_{0}(x)\right)$.

○ For all $i \in[r], x \in X_{i}^{\prime}, v \in N_{B_{i}^{\prime}}(x)$, and distinct $x_{0}, x_{0}^{\prime} \in N_{H^{\prime}}(x) \cap X_{0}^{\prime}$, we have $c\left(\psi_{0}\left(x_{0}\right) v\right) \neq$ $c\left(\psi_{0}\left(x_{0}^{\prime}\right) v\right)$ because $\psi_{0}\left(x_{0}\right)$ and $\psi_{0}\left(x_{0}^{\prime}\right)$ belong to different clusters of $\left(V_{i}\right)_{i \in[r]}$ and $c$ is colour-split with respect to $\left(V_{i}\right)_{i \in[r]}$.

Hence, we can finally apply Lemma 4.7 as follows:

\begin{tabular}{r||c|c|c|c|c|c|c|c|c} 
Parameter & $n_{B}$ & $\varepsilon_{r+1}$ & $\mu^{1 / 2}$ & $d_{B}$ & $d_{B}^{r}$ & $r$ & $r-1$ & $\mathcal{B}^{\prime}$ & $\left(B_{i}^{\prime}\left[X_{i}^{\prime}, V_{i}^{\prime}\right]\right)_{i \in[r]}$ \\
\hline Plays the role of & $n$ & $\varepsilon$ & $\mu$ & $d_{G}$ & $d_{A}$ & $r$ & $\Delta$ & $\mathcal{B}$ & $\left(A_{i}\right)_{i \in[r]}$
\end{tabular}

This yields a rainbow embedding $\psi$ of $H^{\prime}$ into $G^{\prime}$ that extends $\psi_{0}$, such that $\psi(x) \in N_{B_{i}^{\prime}}(x)$ for all $i \in[r]$ and $x \in X_{i}^{\prime}$. Since the colours of $c$ restricted to $G^{\prime} \subseteq G_{B}$ are distinct from the colours already used by $\psi_{0}$, it holds that $\psi$ is a valid rainbow embedding of $H$ into $G$. This completes the proof.

Acknowledgements. The research leading to these results was partially supported by the EPSRC, grant nos. EP/N019504/1 (S. Glock) and by the Deutsche Forschungsgemeinschaft (DFG, German Research Foundation) - 339933727 (F. Joos).

Conflict of Interest: None. 


\section{References}

[1] A. Adamaszek, P. Allen, C. Grosu, and J. Hladký, 'Almost all trees are almost graceful', Random Structures Algorithms 56 (2020), 948-987.

[2] M. Albert, A. Frieze, and B. Reed, 'Multicoloured Hamilton cycles', Electron. J. Combin. 2 (1995), Art. 10, 13 pages.

[3] P. Allen, J. Böttcher, D. Clemens, and A. Taraz, 'Perfectly packing graphs with bounded degeneracy and many leaves', arXiv: 1906.11558 (2019).

[4] P. Allen, J. Böttcher, H. Hàn, Y. Kohayakawa, and Y. Person, 'Blow-up lemmas for sparse graphs', arXiv:1612.00622 (2016).

[5] P. Allen, J. Böttcher, J. Hladký, and D. Piguet, 'Packing degenerate graphs', Adv. Math. 354 (2019), 106739.

[6] J. Böttcher, Y. Kohayakawa, and A. Procacci, 'Properly coloured copies and rainbow copies of large graphs with small maximum degree', Random Structures Algorithms 40 (2012), 425-436.

[7] J. Böttcher, Y. Kohayakawa, A. Taraz, and A. Würfl, 'An extension of the blow-up lemma to arrangeable graphs', SIAM J. Discrete Math. 29 (2015), 962-1001.

[8] J. Böttcher, M. Schacht, and A. Taraz, 'Proof of the bandwidth conjecture of Bollobás and Komlós', Math. Ann. 343 (2009), 175-205.

[9] M. Coulson and G. Perarnau, 'Rainbow matchings in Dirac bipartite graphs', Random Structures Algorithms 55 (2019), 271-289.

[10] B. Csaba, 'On the Bollobás-Eldridge conjecture for bipartite graphs', Combin. Probab. Comput. 16 (2007), 661-691.

[11] M. Drmota and A. Lladó', 'Almost every tree with $m$ edges decomposes $K_{2 m, 2 m}$ ', Combin. Probab. Comput. 23 (2014), $50-65$.

[12] R. A. Duke, H. Lefmann, and V. Rödl, 'A fast approximation algorithm for computing the frequencies of subgraphs in a given graph', SIAM J. Comput. 24 (1995), 598-620.

[13] S. Ehard, S. Glock, and F. Joos, 'Pseudorandom hypergraph matchings', Combin. Probab. Comput. (to appear).

[14] S. Ehard and F. Joos, 'A short proof of the blow-up lemma for approximate decompositions', arXiv:2001.03506 (2020).

[15] P. Erdôs, J. Nešetřil, and V. Rödl, 'On some problems related to partitions of edges of a graph', in Graphs and other combinatorial topics, Teubner-Texte Math. 59 (Teubner, 1983), pp. 54-63.

[16] J. A. Gallian, 'A dynamic survey of graph labeling', Electron. J. Combin. 5 (1998), Dynamic Survey 6, 43.

[17] S. Glock and F. Joos, 'A rainbow blow-up lemma', Random Structures Algorithms 56 (2020), 1031-1069.

[18] S. Glock, F. Joos, J. Kim, D. Kühn, and D. Osthus, 'Resolution of the Oberwolfach problem', arXiv:1806.04644 (2018).

[19] S. Glock, D. Kühn, A. Lo, and D. Osthus, 'The existence of designs via iterative absorption: hypergraph $F$-designs for arbitrary $F^{\prime}$, Mem. Amer. Math. Soc. (to appear).

[20] R. L. Graham and N. J. A. Sloane, 'On additive bases and harmonious graphs', SIAM J. Algebraic Discrete Methods 1 (1980), 382-404.

[21] H.-D. O. F. Gronau, R. C. Mullin, and A. Rosa, 'Orthogonal double covers of complete graphs by trees', Graphs Combin. 13 (1997), 251-262.

[22] A. Hajnal and E. Szemerédi, 'Proof of a conjecture of Erdős', in: Combinatorial Theory and its Applications II, eds. P. Erdôs, A. Rényi, and V.T. Sós, (North Holland, 1970), pp. 601-623.

[23] S. Janson, T. Łuczak, and A. Ruciński, 'Random graphs', Wiley-Intersci. Ser. Discrete Math. Optim. (Wiley-Interscience, 2000).

[24] F. Joos, J. Kim, D. Kühn, and D. Osthus, 'Optimal packings of bounded degree trees', J. Eur. Math. Soc. 21 (2019), 35733647.

[25] P. Keevash, 'A hypergraph blow-up lemma', Random Structures Algorithms 39 (2011), 275-376.

[26] P. Keevash, 'The existence of designs', arXiv:1401.3665 (2014).

[27] P. Keevash, 'The existence of designs II', arXiv:1802.05900 (2018).

[28] J. Kim, D. Kühn, A. Kupavskii, and D. Osthus, 'Rainbow structures in locally bounded colourings of graphs', Random Structures Algorithms 56 (2020), 1171-1204.

[29] J. Kim, D. Kühn, D. Osthus, and M. Tyomkyn, 'A blow-up lemma for approximate decompositions', Trans. Amer. Math. Soc. 371 (2019), 4655-4742.

[30] J. Komlós, G. N. Sárközy, and E. Szemerédi, 'Blow-up lemma’, Combinatorica 17 (1997), 109-123.

[31] J. Komlós, G. N. Sárközy, and E. Szemerédi, 'On the Pósa-Seymour conjecture’, J. Graph Theory 29 (1998), 167-176.

[32] J. Komlós, G. N. Sárközy, and E. Szemerédi, 'Proof of the Seymour conjecture for large graphs', Ann. Comb. 2 (1998), 43-60.

[33] J. Komlós, G. N. Sárközy, and E. Szemerédi, 'Proof of the Alon-Yuster conjecture', Discrete Math. 235 (2001), $255-269$.

[34] A. Kotzig, 'On certain vertex-valuations of finite graphs', Utilitas Math. 4 (1973), 261-290.

[35] D. Kühn and D. Osthus, 'The minimum degree threshold for perfect graph packings', Combinatorica 29 (2009), 65-107.

[36] D. Kühn and D. Osthus, 'Hamilton decompositions of regular expanders: A proof of Kelly's conjecture for large tournaments', Adv. Math. 237 (2013), 62-146.

[37] C. McDiarmid, 'On the method of bounded differences', in Surveys in Combinatorics (Norwich, 1989), London Math. Soc. Lecture Note Ser. 141 (Cambridge Univ. Press, 1989), pp. 148-188.

[38] R. Montgomery, A. Pokrovskiy, and B. Sudakov, 'Decompositions into spanning rainbow structures', Proc. Lond. Math. Soc. 119 (2019), 899-959.

[39] R. Montgomery, A. Pokrovskiy, and B. Sudakov, ‘A proof of Ringel's Conjecture', arXiv:2001.02665 (2020). 
[40] R. Montgomery, A. Pokrovskiy, and B. Sudakov, 'Embedding rainbow trees with applications to graph labelling and decomposition', J. Eur. Math. Soc. 22 (2020), 3101-3132.

[41] V. Rödl, 'On a packing and covering problem', European J. Combin. 6 (1985), 69-78.

[42] V. Rödl and A. Ruciński, 'Perfect matchings in $\epsilon$-regular graphs and the blow-up lemma', Combinatorica 19 (1999), 437452.

[43] A. Rosa, 'On certain valuations of the vertices of a graph', in Theory of Graphs (Internat. Sympos., Rome, 1966) (Gordon and Breach, New York; Dunod, Paris, 1967), pp. 349-355.

[44] A. Żak, 'Harmonious order of graphs', Discrete Math. 309 (2009), 6055-6064. 